\title{
Theodosius' Sphaerica: A Second Arabic Translation
}

\author{
Paul Kunitzsch \\ Davidstrasse I7 \\ 81927 Muenchen, Germany \\ RICHARD LORCH \\ Io Nightingale Court, Leam Terrace \\ Leamington $\mathrm{Spa}, \mathrm{CV}_{3}$ I IDQ, Great Britain \\ (richardlorch@btinternet.com)
}

\begin{abstract}
An Arabic translation of the Sphaerica of Theodosius of Bithynia (around Ioo B.C.) accompanied by its Latin translation by Gerard of Cremona had been edited by P. Kunitzsch and R. Lorch in 20 Io. Apart from that, there exists a second Arabic translation of this text in two manuscripts in Hebrew cursive script. For readers interested in the matter, we here edit the text of Book I of the work in this second version and discuss some differences here from the version edited in 2010.
\end{abstract}

KeYwords: Theodosius of Bithynia, his work Sphaerica, anonymous Arabic translation (edited 20I0), Qusțā ibn Lūqā - second Arabic translation, partly edited and discussed here for comparison.

In 20I0, we have edited an Arabic translation of Theodosius' Sphaerica together with its Latin translation by Gerard of Cremona (Toledo, $2_{2}{ }^{\text {th }}$ century AD). ${ }^{.}$The Arabic version of 2010 has been edited from three manuscripts; it remained anonymous, the translator(s) were not mentioned in the

I. Theodosius, Sphaerica, Arabic and Medieval Latin Translations, Edited by Paul Kunitzsch and Richard Lorch, Stuttgart: Franz Steiner Verlag, 20 Io (Series Boethius, Vol.62), 43I pp. In the present article, this will shortly be quoted as: 20I0:x (= page number), y (= line number). Literature quoted there (cf. Bibliography pp. 429-43I) will here be quoted in the abbreviated form used there. Literature published after 20 Io will of course be quoted here with full title etc.

Kunitzsch, Paul; Lorch, Richard (2018-2019). «Theodosius' Sphaerica: A Second Arabic Translation». Suhayl I6-I7, pp. I 2 I-I48. ISSN: I 576-9372. DOI: Io.I344/SUHAYL20I9.I6-I 7.4. 
sources (cf. 20I0:2f.). In addition, R. Lorch found two manuscripts (in Hebrew cursive script) containing another Arabic version of the Sphaeri$c a^{2}$ of which we here edit the text of Book I, transformed into Arabic script, in order to give interested readers an idea of the form and content of this second version of the text.

This second Arabic translation of the Sphaerica has been found in two manuscripts in cursive Hebrew script. In both manuscripts the translation is ascribed to Qusțā ibn Lūqā (d. ca. 300 H/9I2-I3 AD). The two manuscripts are:

$$
\begin{aligned}
& \mathbf{F}=\text { Florence, Biblioteca Medicea Laurenziana, Orientali I24, 76ff.; and } \\
& \mathbf{C}=\text { Cambridge, University Library, Add. I220, ff. Ir-50r. }
\end{aligned}
$$

The script, a cursive form of Hebrew, in $\mathbf{F}$ is rather clearly readable, whereas in $\mathbf{C}$ the script often appears quite hastily and carelessly made, also it seems that the pens and/or the hands of writers had several times changed. The language is Arabic, but in a very colloquial form (judeoArabic?); for our present rendering we transformed it into normal written Arabic (with the deviant colloquial Hebrew variants only shown in the apparatus of Propos. I, in order to save the space for the rest of the text). So, as it seems, this text was produced around 900AD in Baghdad, then somehow reached al-Andalus, i.e the Arabic-dominated zones of Spain, where in the late period it came into the hands of Jewish inhabitants of the area who then transformed it somehow, perhaps from an Arabic manuscript in the Andalusian-Maghrebi ductus of the Arabic script and with the spoken help of some Arabs (in the colloquial dialect), into their contemporary judeo-Arabic language and writing.

2. Cf. Lorch [ 1996], p. I64f.; idem, The "Second" Arabic translation of Theodosius' Sphaerica, in: From Alexandria, Through Baghdad. Surveys and Studies... in Honor of J.L. Berggren, ed. N. Sidoli - G. Van Brummelen, Berlin - Heidelberg: Springer Verlag, 20 I4, pp. 255-258. 


\section{Some Comments}

Generally the mathematical contents and proceedings in the second translation are the same as in 20I0, apart from the fact that here often other vocabulary and another style of expression is used. As for the diagrams of the propositions, in our photocopies of $\mathbf{F}$ and $\mathbf{C}$ they were mostly not clearly visible, but we received coloured scans of $\mathbf{C}$ in which the diagrams were well visible. ${ }^{3}$ The letters in the diagrams here (in Book I) reach from $A$ to $N$ (following the Greek alphabet, and in Arabic the abjad series, omitting $U$ and $I$ ). ${ }^{4}$ In the following some differences in $\mathbf{F C}$ from 20 IO will shortly be mentioned. But not all such differences must be due to the translation or transmission of the second Arabic version, it could well be that its translator Qusțā ibn Lūqā used a Greek source that itself offered readings etc. different from those in the source of the translation of 2010.

Propos. I, 4: In 20I0: $23-27$ both in the diagram and in the corresponding mathematical text 9 diagram letters are used, $A-L$, with the omission of $H$. The same is the case in the second translation, but here one more letter is omitted: $T$, also both in the diagram and in the text, i.e. altogether only 8 and not 9 letters.

Propos. I, 6, lines 35-36: The sentence between the pointed brackets (wa-khatt $K N \ldots$ ila $m u h \bar{t} t i h \bar{a}$ ) is omitted in $\mathbf{F C}$; it has been added from 20I0: $34,45-46$ (= Latin p. 35, 58-59), because there and in the Greek text it continues and completes the preceding sentence about line $T L$.

Propos. I,7: The order of the six letters, $A-Z$, and accordingly the mathematical discussion in 2010 and in the second translation are somewhat different, but the mathematical results of the two are equal.

Propos. I, I3, lines 7 and 9: The diagram and the text of this proposition contain 9 diagram letters, $A-K$ in the normal alphabetical order (so in the Greek text and in 20I0, line 8: khatt $H K$, and lines I I and I2, i.e. twice, the same); here the second translation has in all places instead of $K$ the letter $L$ (lines 7 and 9 -here twice).

3. We use this moment to express our graditude to Prof. M. Folkerts (Munich), Prof. Dj. Paunić (Novi Sad), Dr. B. van Dalen (Munich) and Dr. Sonja Brentjes (Berlin), who helped us to obtain the scans of manuscript $\mathrm{C}$ from Cambridge and to transform the edition into the Suhayl format.

4. Cf. the table in 20Io: 8. 
Propos. I, I4, line Io: ' $a m \bar{u} d H T$, here the Greek text and 20I0: 56, Io have the letters TA. From line I I on the two versions, 20 IO and the second translation, show several differences in the text, but use the same letters in the argumentation and at the end arrive at the same result. The arrangement of the diagrams and their letters in the two versions is also slightly different, but indicates the same mathematical effect.

Propositions I, I5 and I6: The diagrams of these two propositions are identical with the diagram of Propos. I4, both in 2010 and in the second translation.

Propos. I, I7: The diagram of this proposition has the same form in both versions. But of the six letters in that diagram the positions of two letters were exchanged: the place of $A$ in 2010 is taken by $B$ in the second translation, and the place of $B$ in 2010 by $A$ in the second translation. The text of the proof is nearly identical in the two versions and arrives at the same result.

Propos. I, I8: In this proposition, the difference of both diagram and text in the two versions is remarkably greater. The diagram (and the text) in 20 Io have only 5 letters, $A-E$, whereas the second translation has in both instances 6 letters, $A-Z$ (there is no $Z$ in 20I0). The way of the proof is similar in the two versions until line 8 (second translation)/ 20I0: 68,9; hereafter they differ in general and in details, the second translation then introducing the additional letter $Z$. At the end, the final conclusion sounds almost identical in both versions.

Propos. I, 20: This proposition contains the diagram in two parts: the larger part shows two circles, marked with 5 letters, $A, B, G, D, K$, and separately a smaller detail of that diagram, with 4 letters, $E, Z, H, T$ (so in the Greek text and in 2010: 72-76). The second translation has mainly the same forms of the diagrams and the same mathematical descriptions with one exception: both at the lower end of the diameter of circle $A B D$ in the bigger diagram and in the pertaining text (in lines 9, IO, I I, I4 and 20) instead of the letter $K$ in 2010 etc. it has $T$, a second time beside its proper place in the smaller diagram, there together with $E, Z$ and $H$. So the second translation uses $T$ also in the bigger diagram, in combination with its letters $A, B, G$ and $D$. Nevertheless, the proof of the second translation comes to the same result as 20I0: 20-2I, that line $A K(A T)$ is equal to line $E T$, and line $A K(A T)$ is the diameter of the sphere, and therefore line $E T$ is equal to the diameter of the known sphere. The reason for using $T$ a second time 
(instead of $K$ ) in the two manuscripts of the second translation could be a simple misunderstanding of an Arabic source manuscript in the Andalusian-Maghrebi ductus: in that style of writing these two letters can look quite similar, and when written a bit carelessly and then read and reproduced also somewhat carelessly and without precise knowledge of the matter and the background, such a confusion between $T$ and $K$ might easily happen.

Propos. I, 2 I: The figure of the diagram in this proposition is differently formed in the Greek text, in 20 Io and here in the second translation. In the Greek text and in 20 Io it has 8 letters, $A-T$; in the second translation it has only 6 letters, $A, B, G, D, E$ and $T ; Z$ and $H$ are here missing, both in the diagram and in the mathematical text. Therefore the mathematical description in the two versions is only partially identical: from 20 I0: 78,8 = second translation line 6 on it has a different argumentation using the different letters.

So far our comments on the differences in Theodosius' Sphaerica between the edition of 20 IO and the second Arabic translation. As already said, we have transformed the colloquial judeo-Arabic dialect into standard written Arabic form (citing the colloquial variants in the apparatus only in Propos. I). Further, in the course of the text, we have only quoted variants from the manuscripts when they might influence the technical meaning of a sentence. In any way, of historical importance was "20Io", the (first) Arabic version circulating and being widely cited in the Orient, and in the same also in the Latin West through Gerard of Cremona's Latin translation of the $12^{\text {th }}$ century. This present edition is only meant to give interested readers an idea of what there was further produced, in addition to the well-known "standard version" edited in 20 Io. 


$$
\text { كتاب ثاوذوسيوس في الكرة ترجمة قسطا بن لوقا }
$$

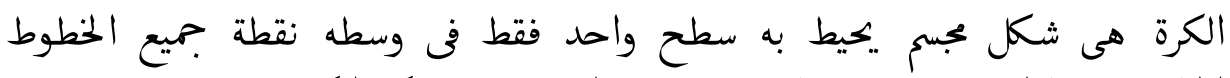

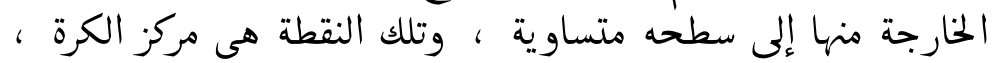

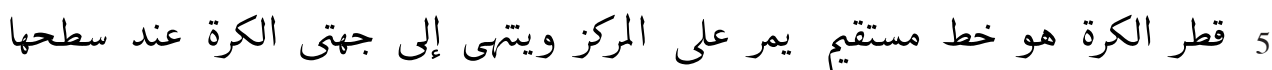

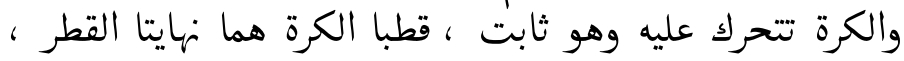
وقطب الدائرة على الكرة هى نقطة على سطح الكرة جميع الخطوط الخارجة منها إلى تلك الدائرة متساوية ،

الدوائر التى تخط على الكرة يقال بعدها من المركز سواء إذا كانت الأعمدة الخارجة

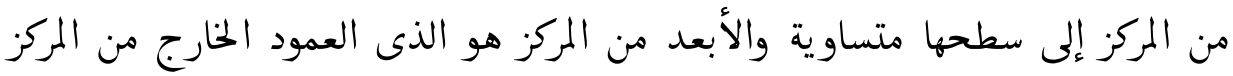
IO

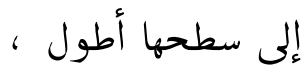

والسطح يقال إنه منحرف على السطح إذا كانت الخطوط الخارجة في السطحين

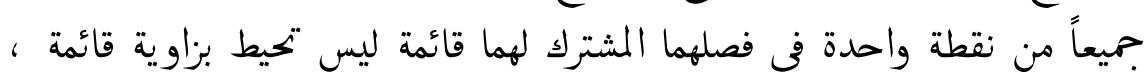

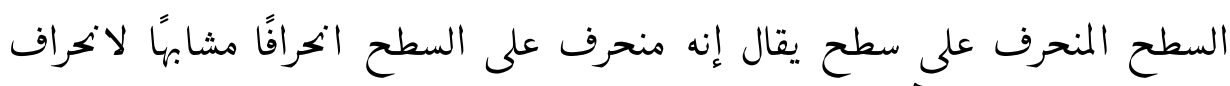

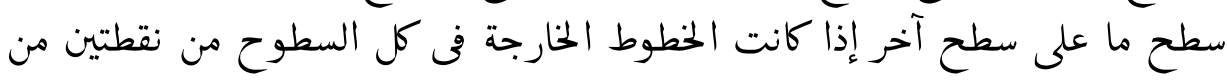

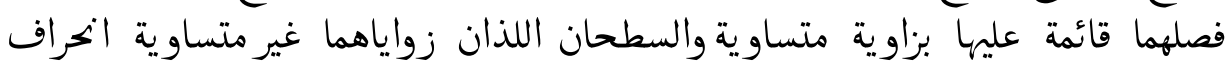

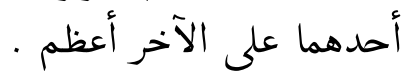

[1] إذا كان يفصل سطح ما بسيط كرة فإن فصله الذى يكون فى بسيط الكرة هو يحيط

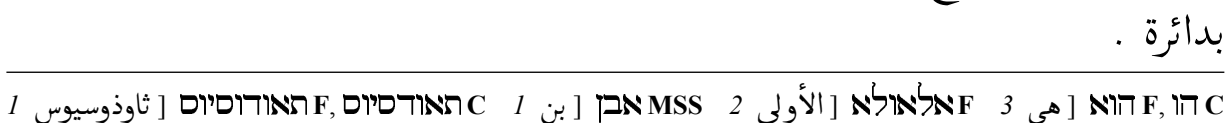

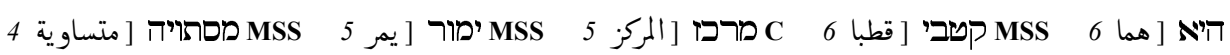

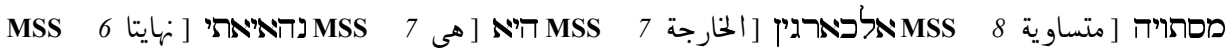

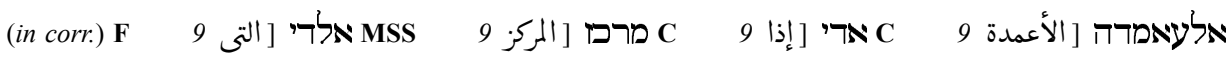

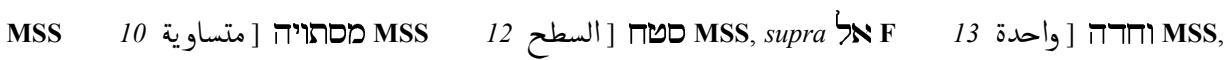

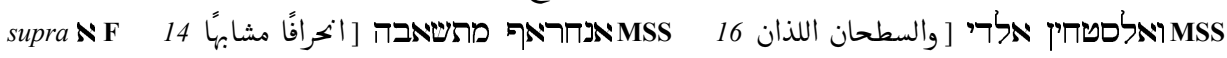
16 MSS 


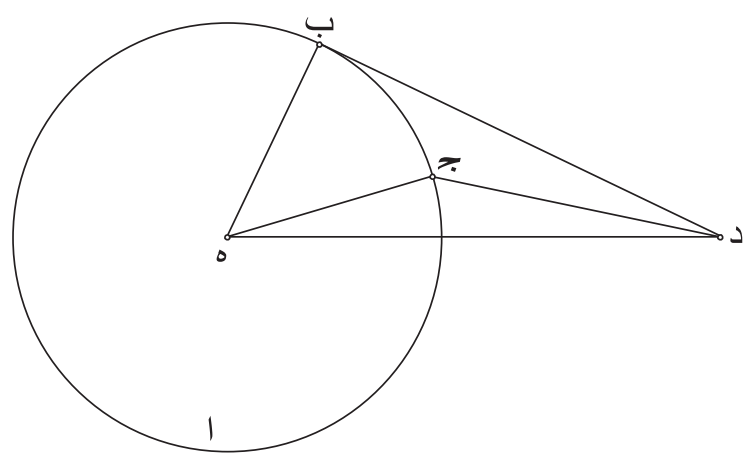

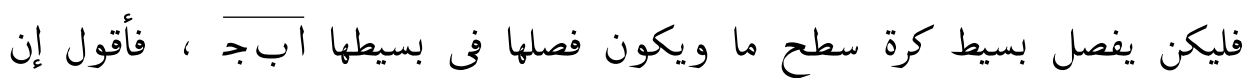

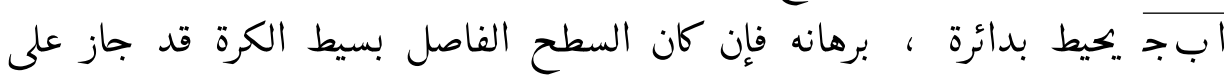

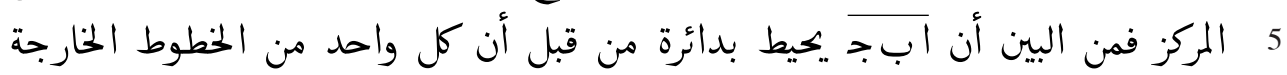

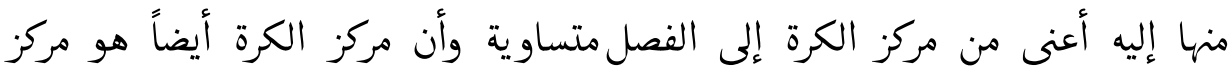

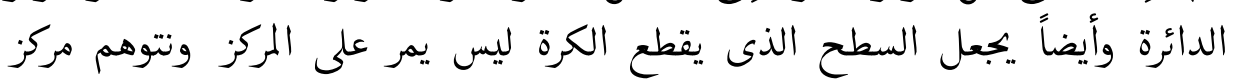

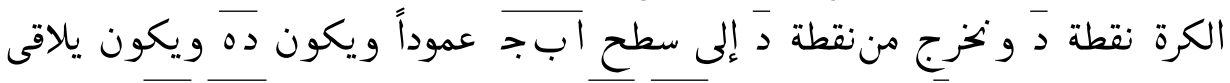

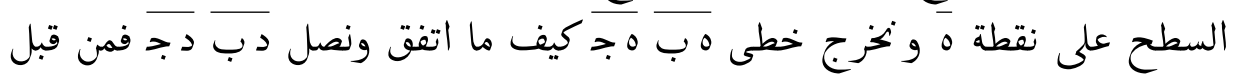

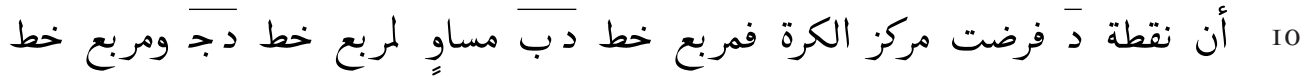

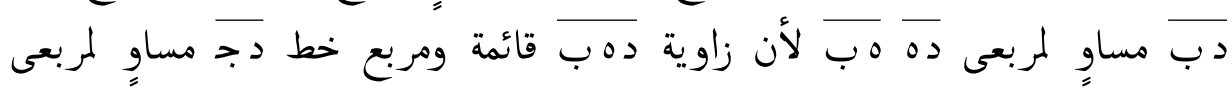
خطى دانٍ

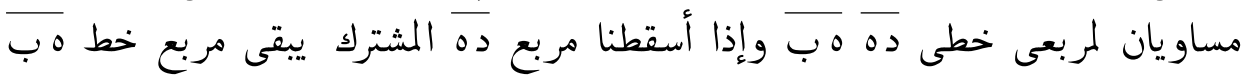

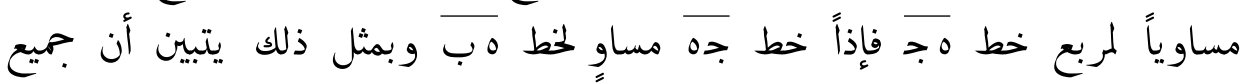

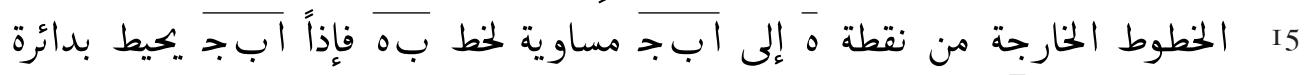

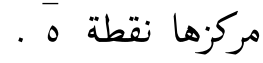

وهنالك استبان أن إذا أخرج من مركز الكرة إلى سطح الدائرة من الدوائر التى عليها

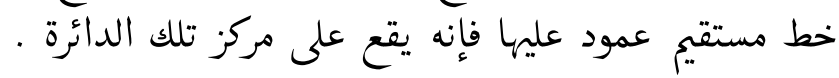

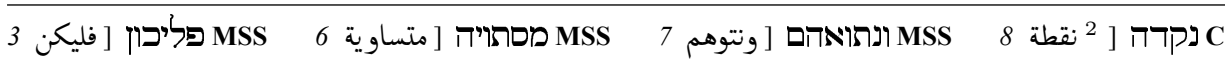

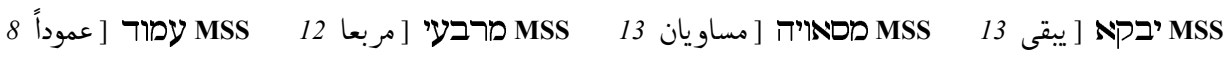

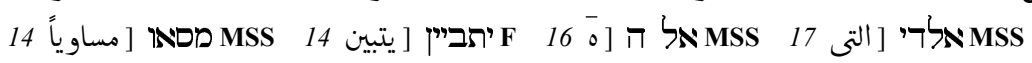




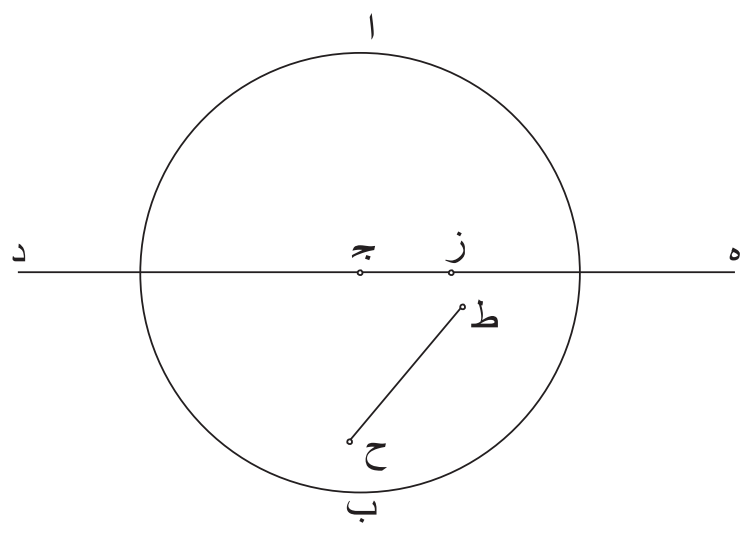

فلتوهم الكرة المفروضة التى نريد أن نجد مركزها يفصلها سطح ما ففصله يكون

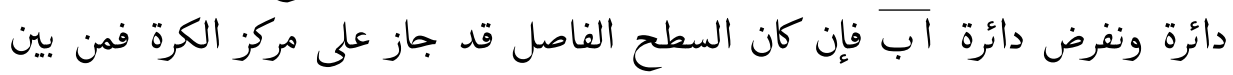

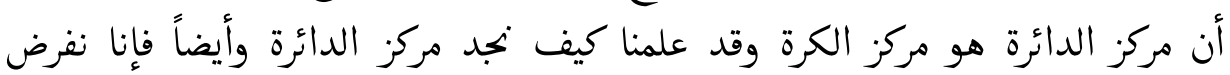

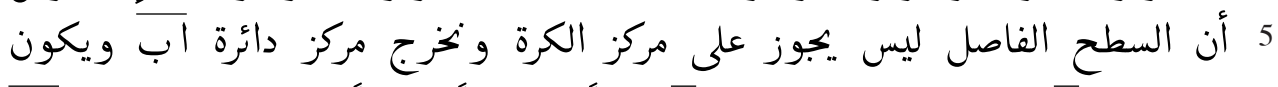

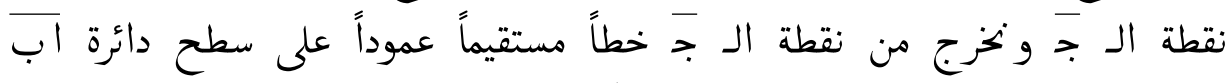

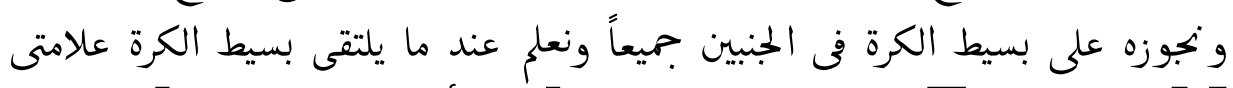

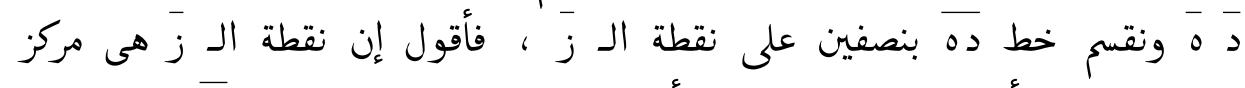

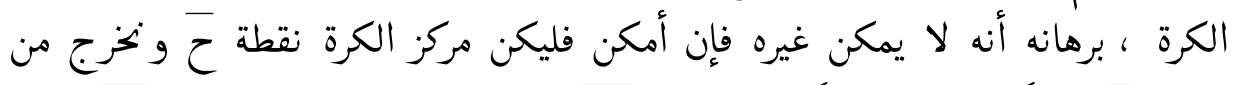

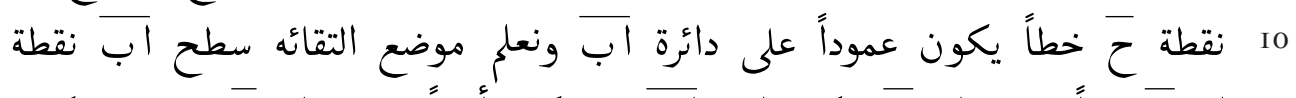

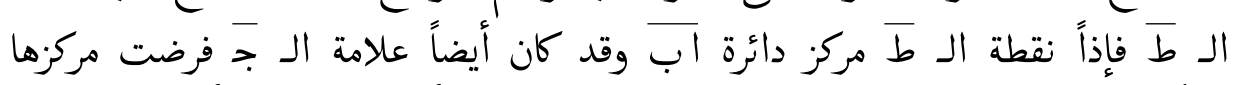

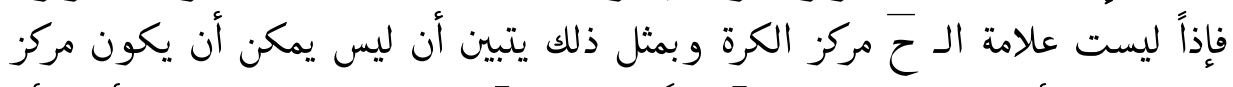

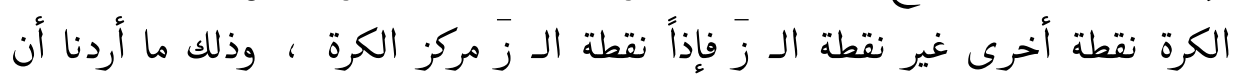

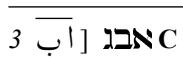


[ب] [إذا ماست كرة سطحاً ما وليس يفصلها فإنها لا تماسه إلا على نقطة واحدة .

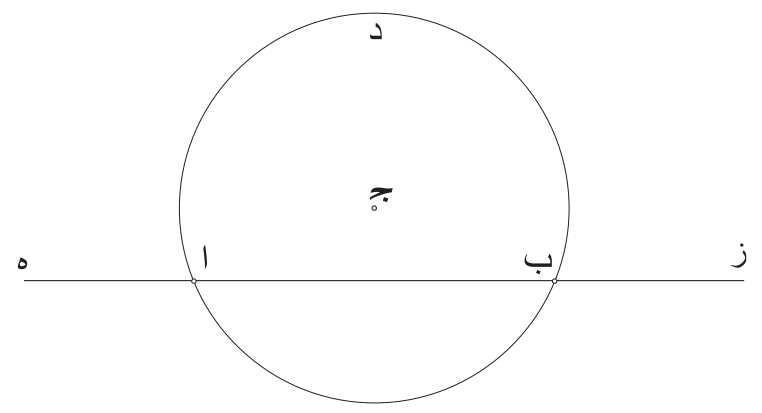

فإن أمكن أقول إنها تماس سطحاً على نقطتى آ بَ ونخرج على نقطتى آ بَ سطحاً

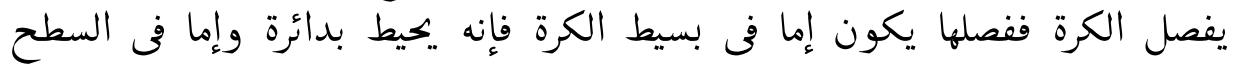

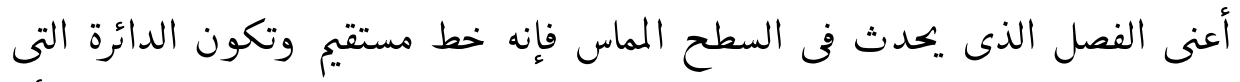

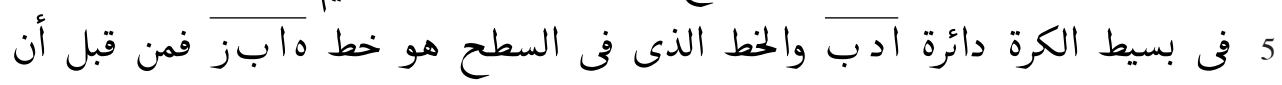

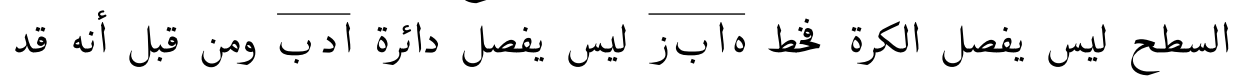

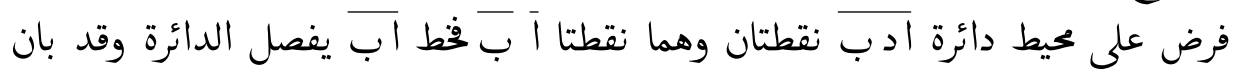

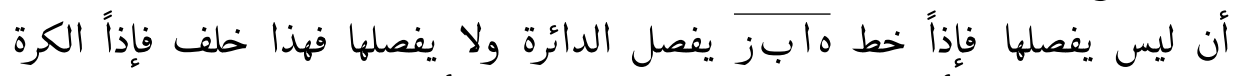

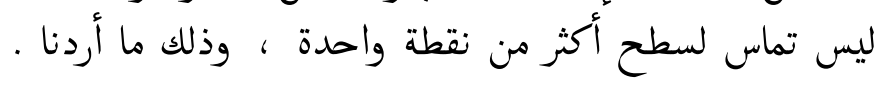

[ع] إذا ماست كرة سطحاً ما وليس يفصلها فإن الخط الخارج من مركز الكرة إلى نقطة

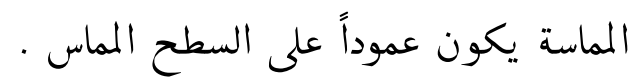

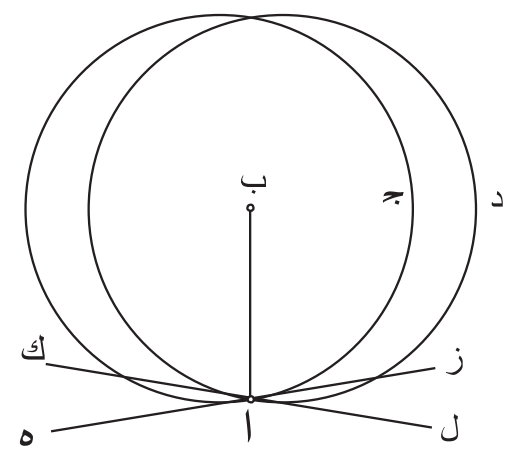

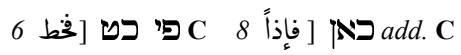


مثال ذلك نتوهم كرة تماس سطحاً ما وليس يفصلها والمماسة على نقطة آ ومركز الكرة

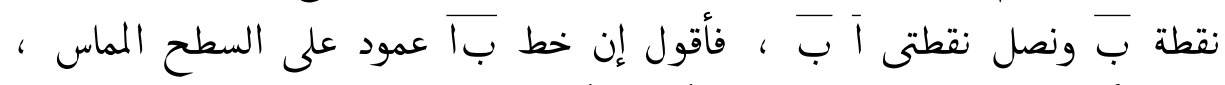

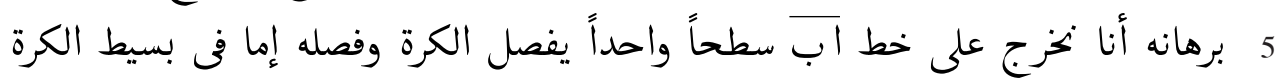

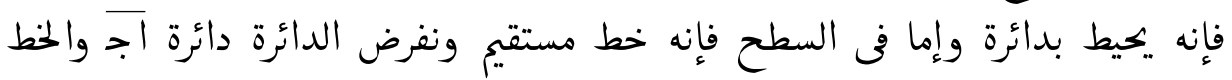

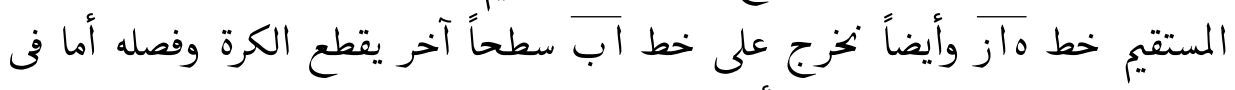

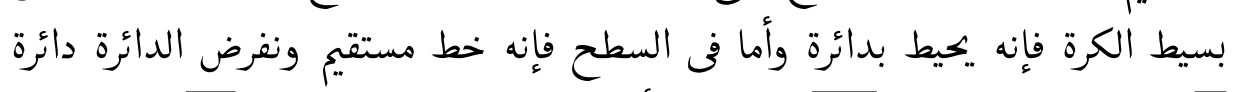

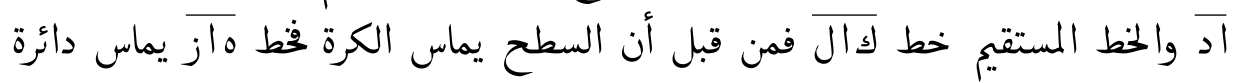
IO

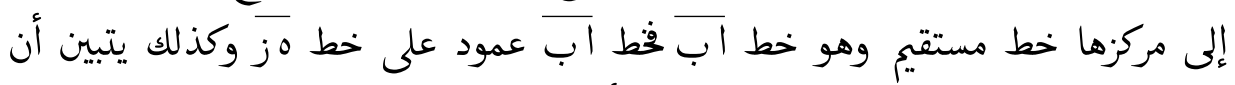

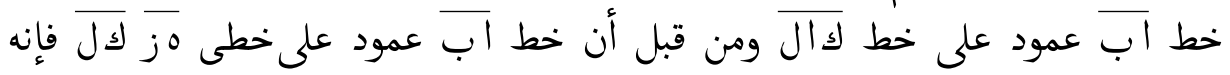

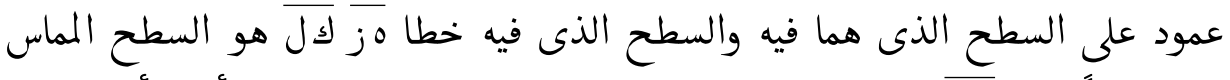

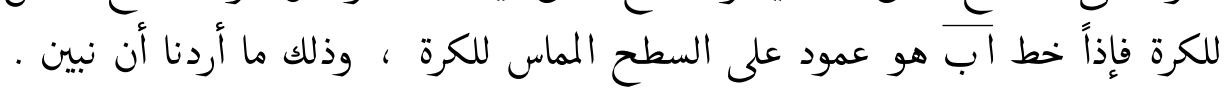

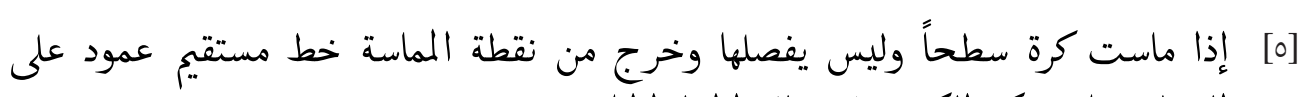

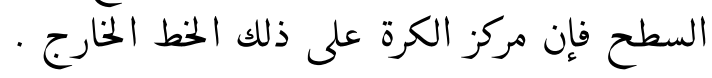

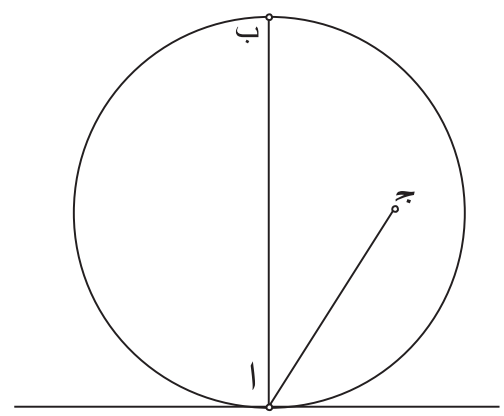

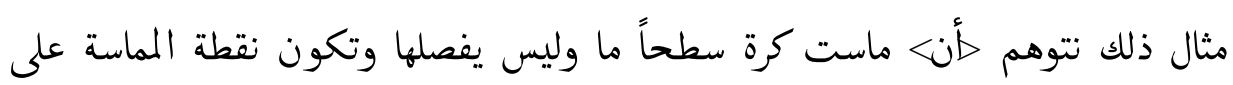

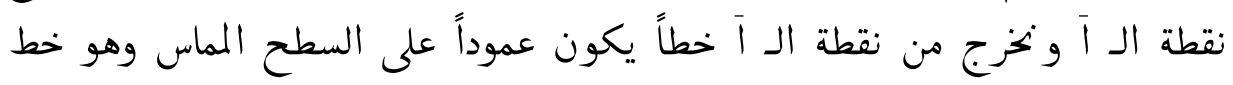
בט [خطى MSS 


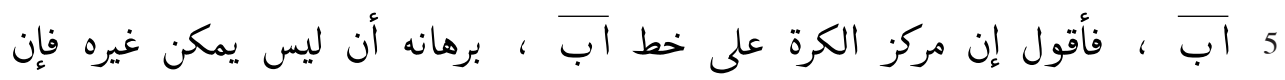

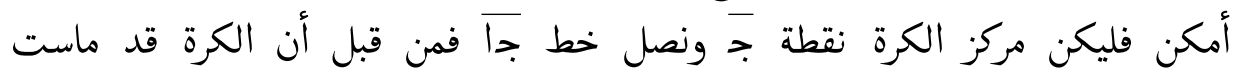

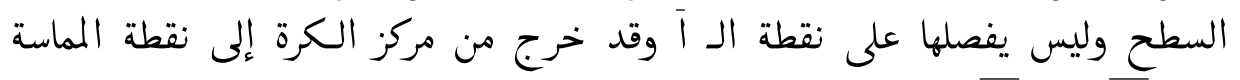

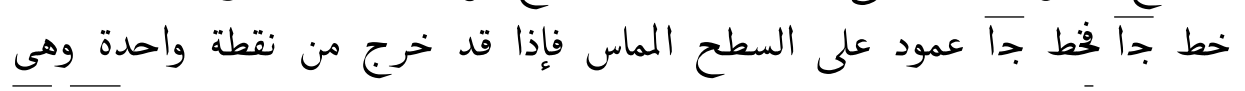

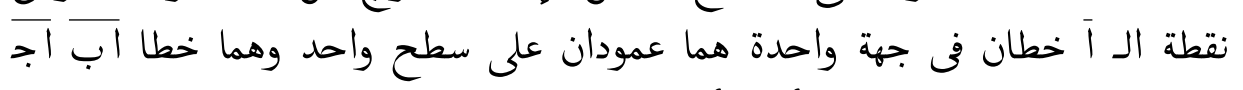

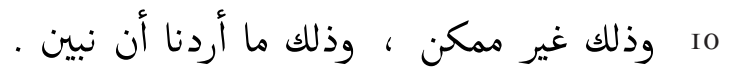

الدوائر التى على الكرة ما كان منها على مركز الكرة هى دوائر عظام وما كان منها

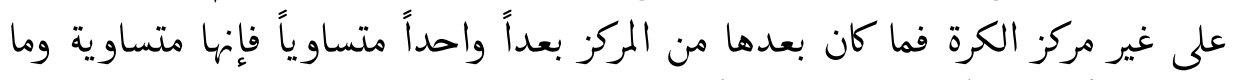

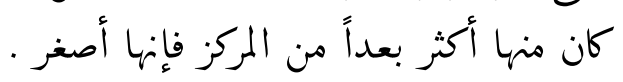

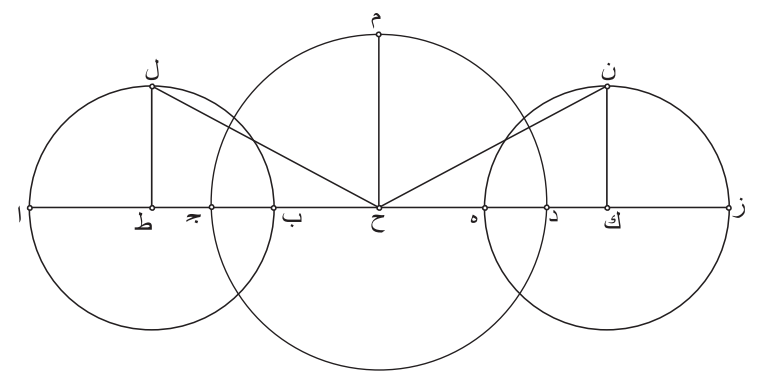

مثاله أنا نفرض دوائر اب جد مز ولتكن دائرة جد جائزة على مركز الكرة ودائرتا

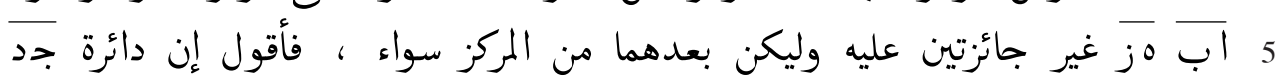

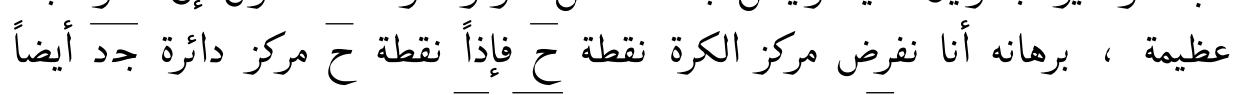

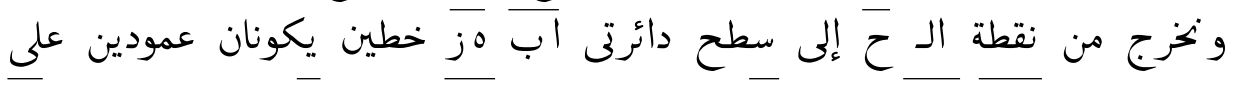

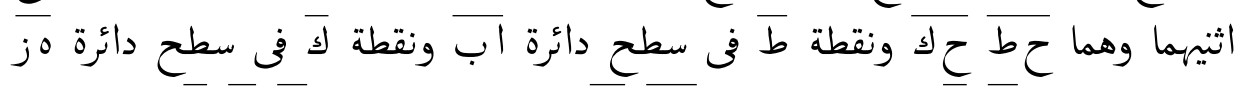

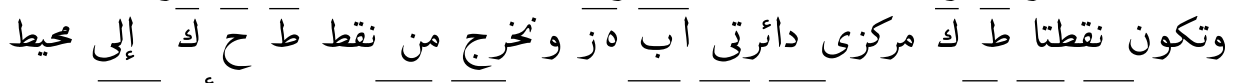

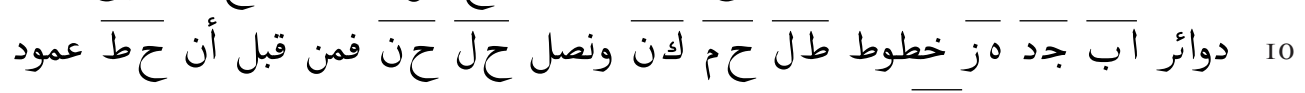

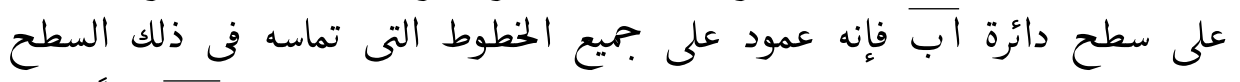

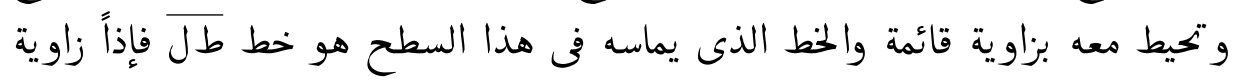
ل لطاح 


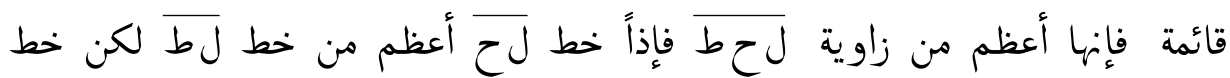

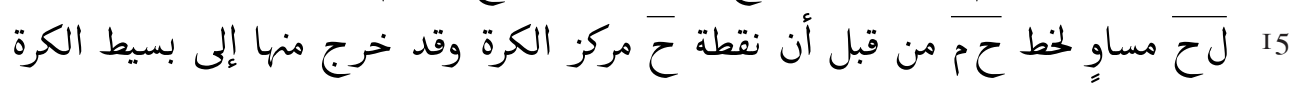

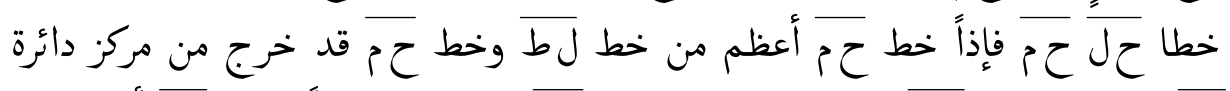

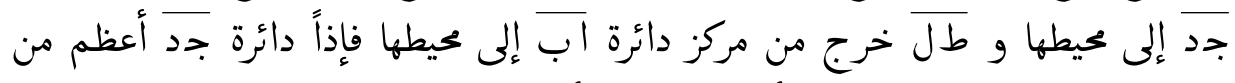

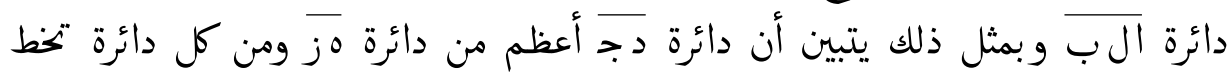

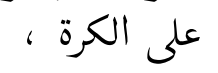

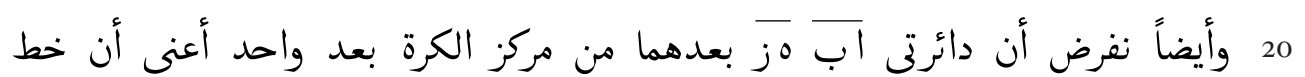

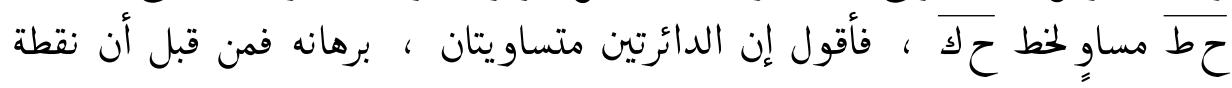

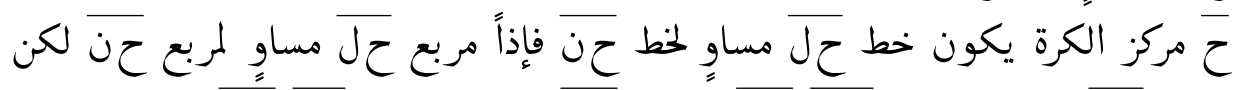

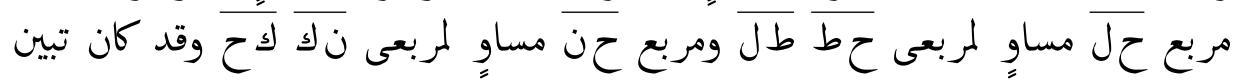

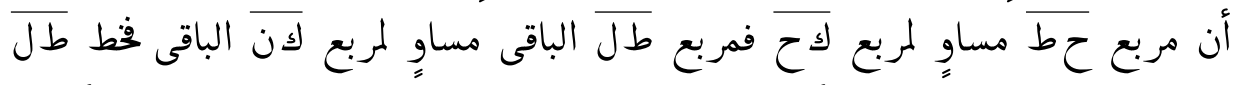

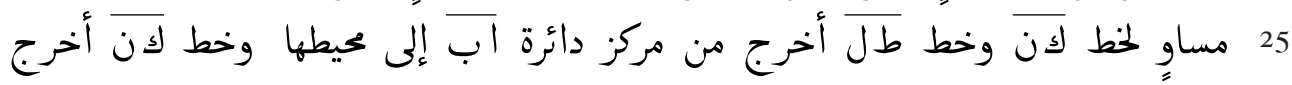

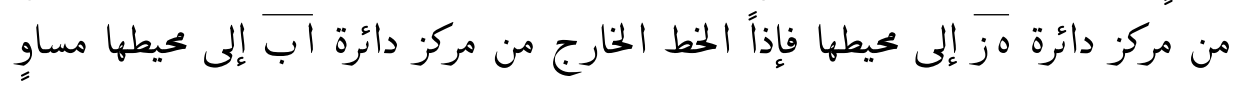

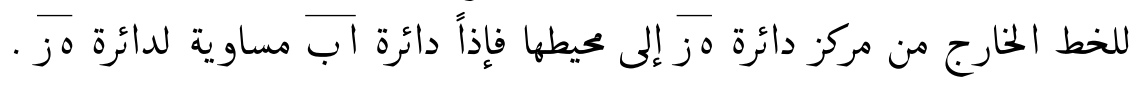

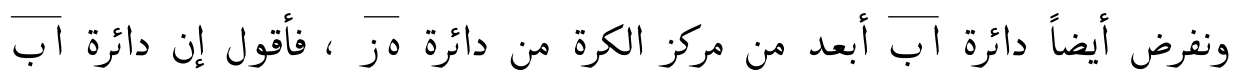

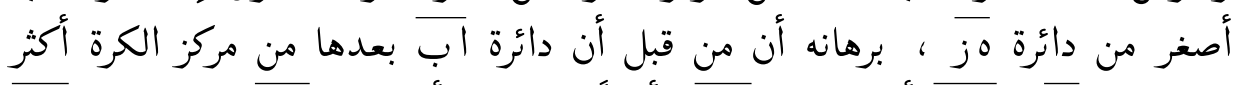

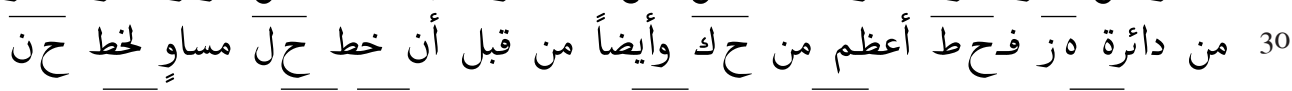

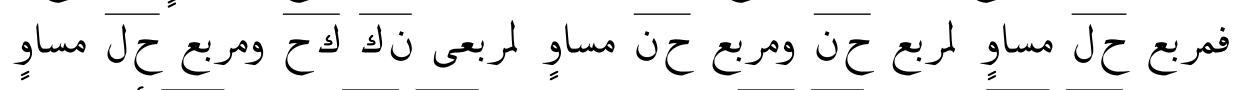

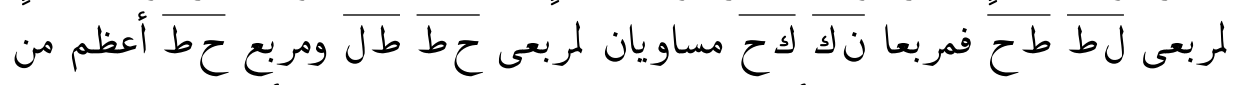

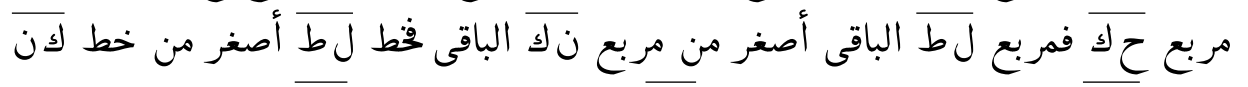

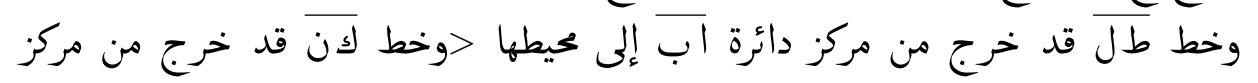

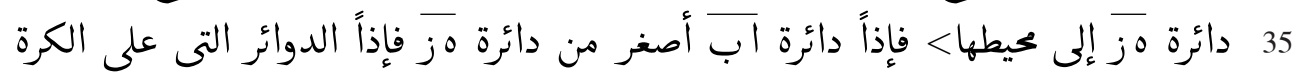

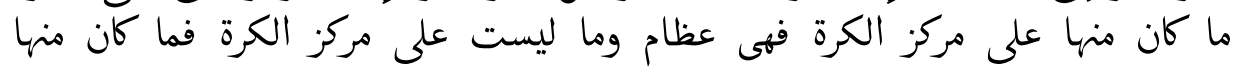

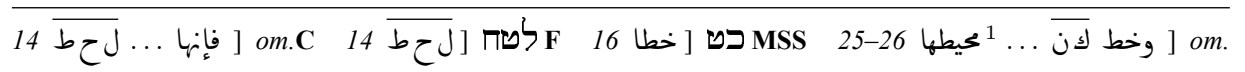

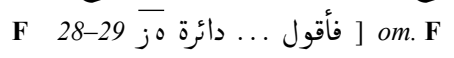


بعدها من المركز بعداً سواء فهى متساوية وما كان بعدها أكثر فهى أصغر ، وذلك ما

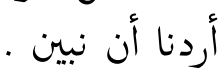

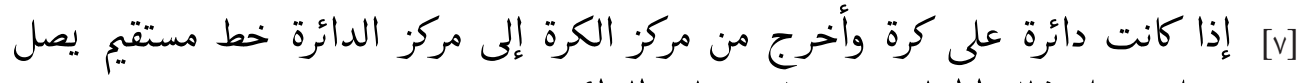
فيما بينها فذلك الخط عمود على سطح الدائرة من مركن الكرة إلى

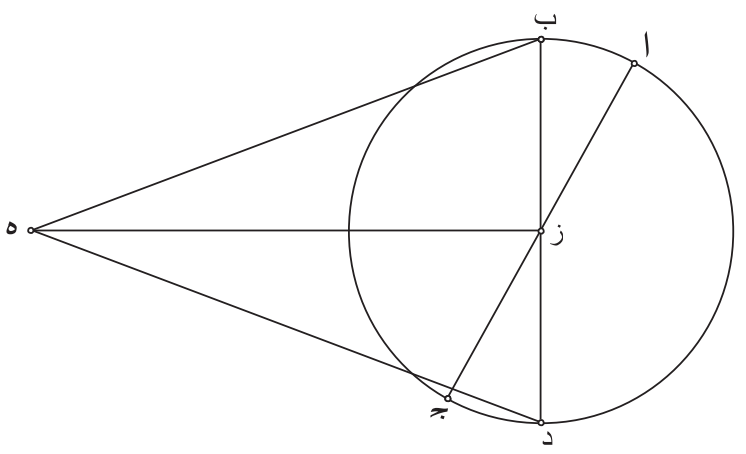

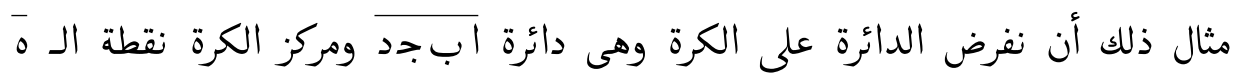

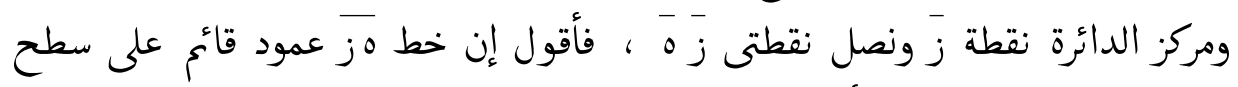

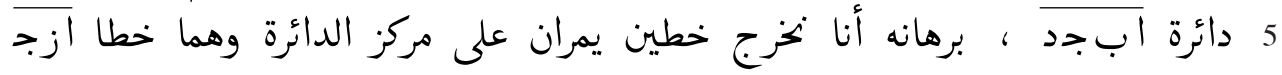

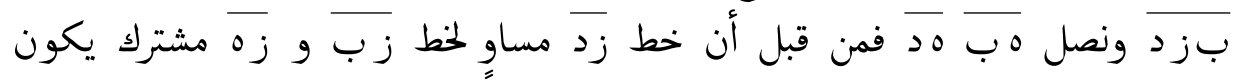

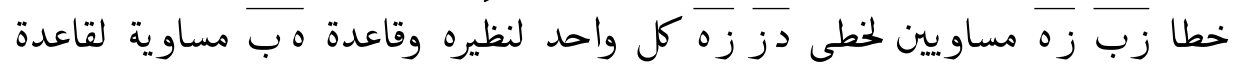

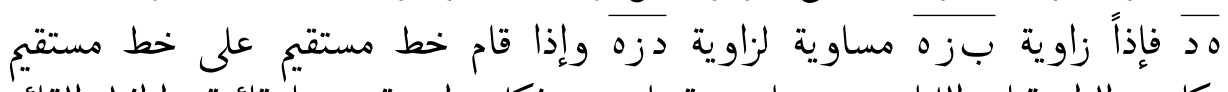

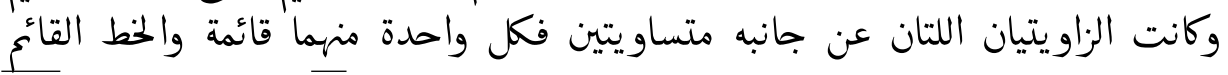

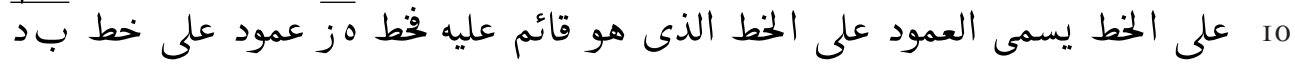

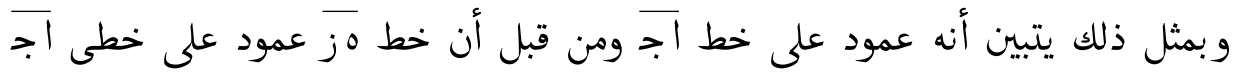

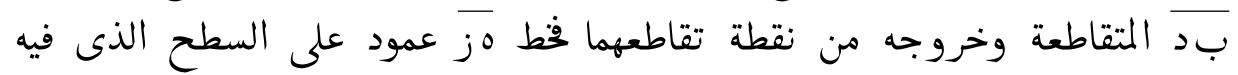

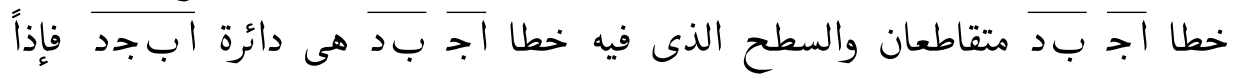

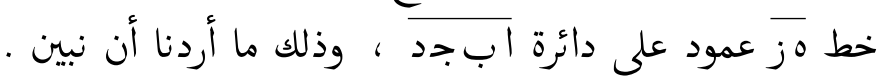

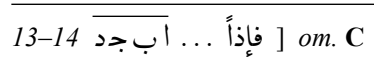


[^] إذا كانت دائرة على كرة وأخرج من مركز الكرة إلى سطح الدائرة عمود ومر فى الجنبين مهيعاً إلى بسيط الكرة فإنه يقع على قطبى تلك الدائرة .

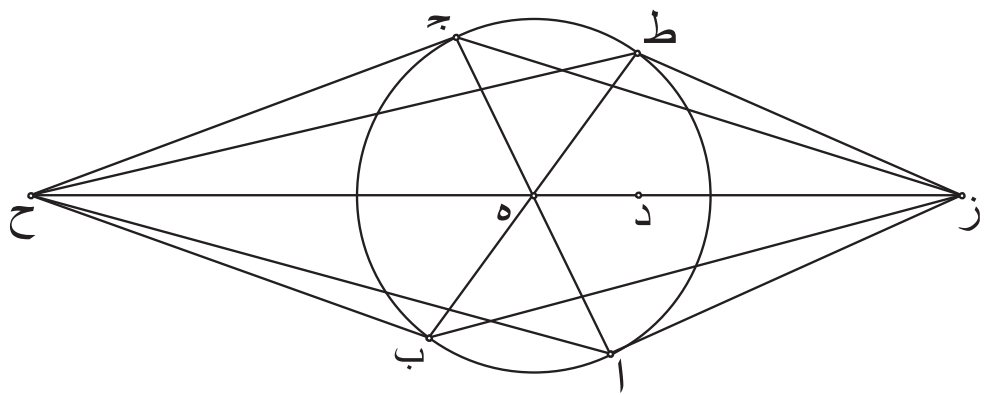

مثال ذلك أنا نفرض دائرة على كرة وهى دائرة اب ج ونفرض مركز الكرة على نقطة

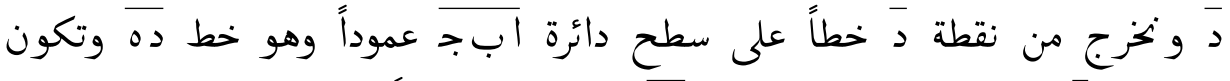
5 نقطة الـ هَ مركز الدائرة و نخرج خط ده في الجهتين جميعاً ونعلم موضع التقائه بسيط

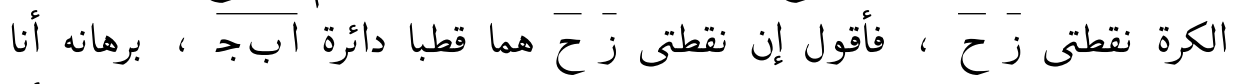

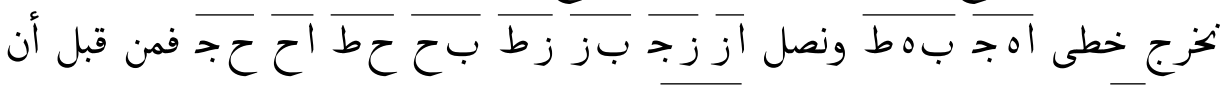

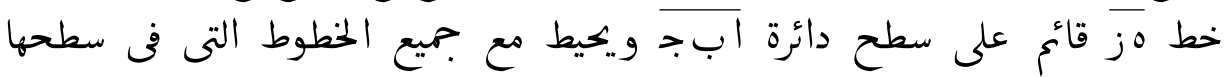

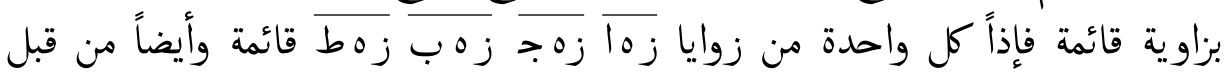

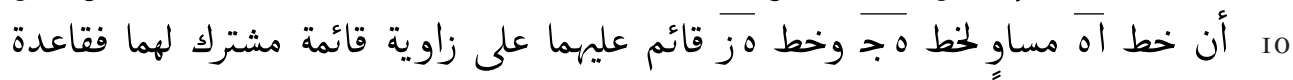

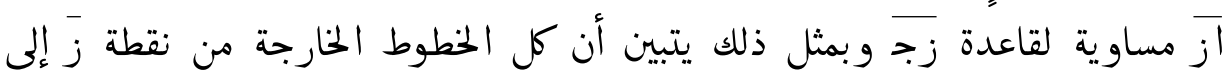

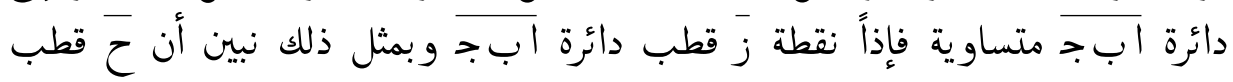

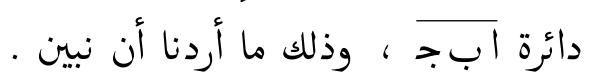

[9] إذا كانت دائرة على كرة وأخرج من واحد قطييها خط إلى مركز الدائرة كان ذلك

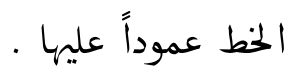
والعمل في بيان ذلك الشكل كالعمل في بيان الشكل الذى قبل ذلك ، وذلك ما

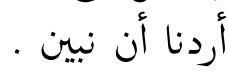


[.1] إذا كانت دائرة على كرة وأخرج من أحد قطيها خط مستقيه عمود عليها فإنه يقع على مركزها وإن أخرج الخط إلى جهته الأخرى فإنه يقع على فئل قطبها الآخر .

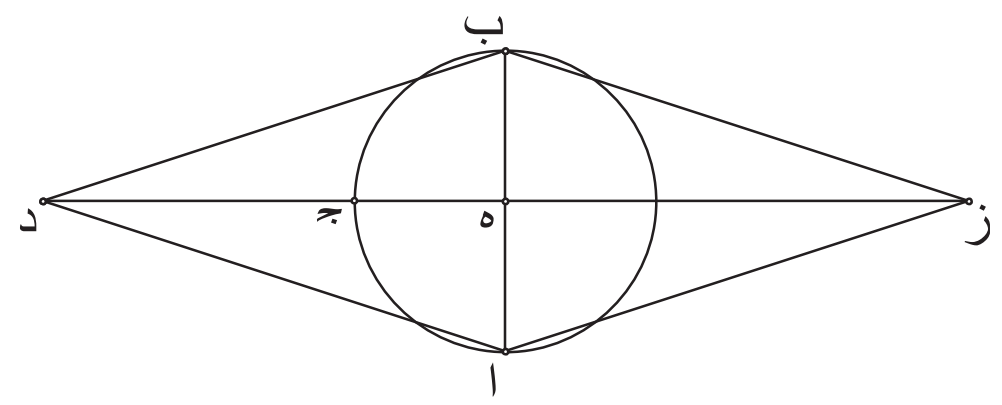

مثال ذلك أنا نفرض دائرة على كرة وهى دائرة ابجاج ونخرج من أحد قطيها وهو

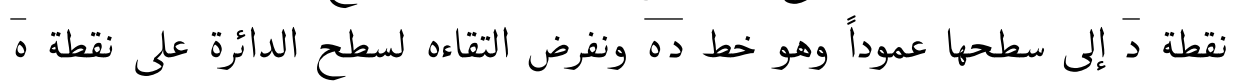

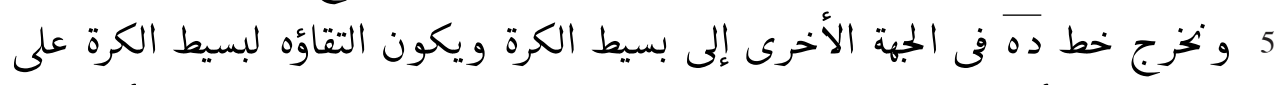

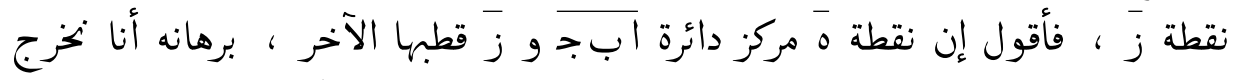

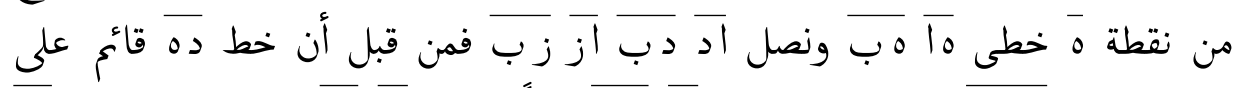

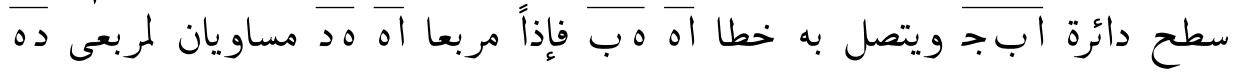

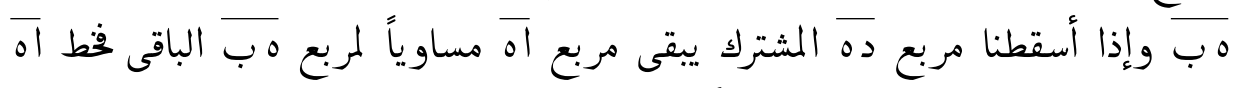

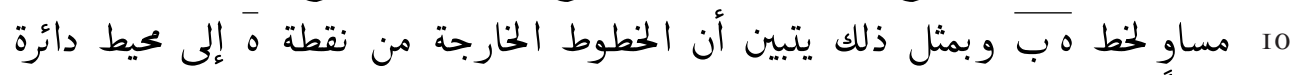

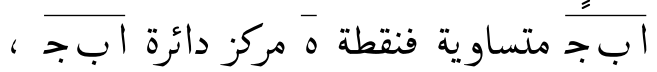

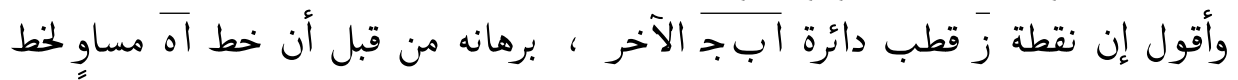

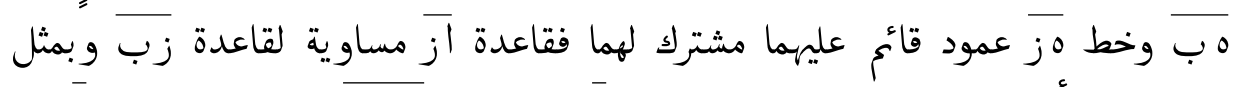

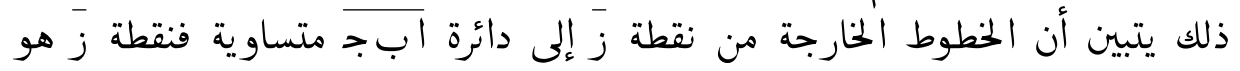

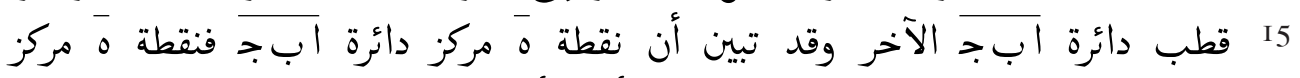

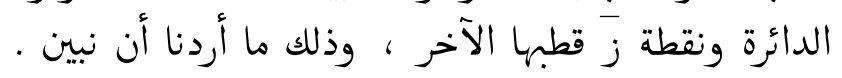

[11] إذا كانت دائرة على كرة وأجيز على قطيها خط مستقيم فإن ذلك الخط يكون عموداً عليها ويمر على مركزها ومركز الكرة . 


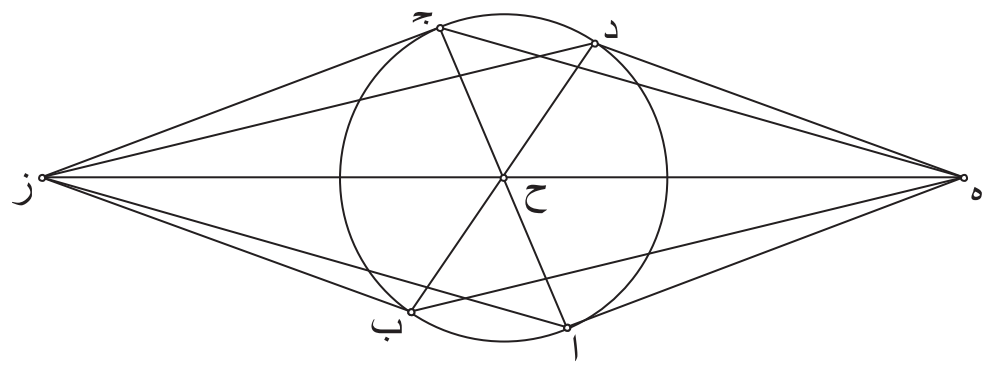

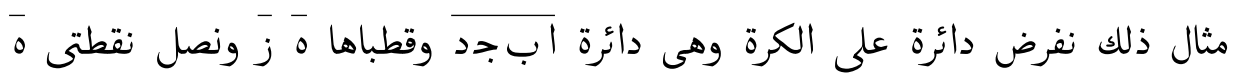

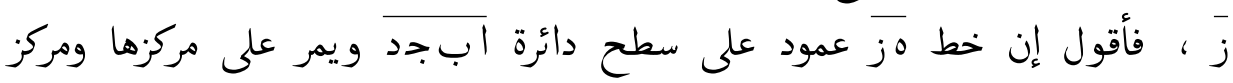

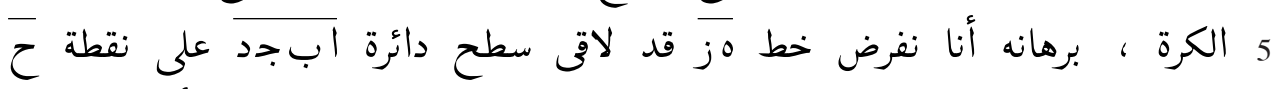

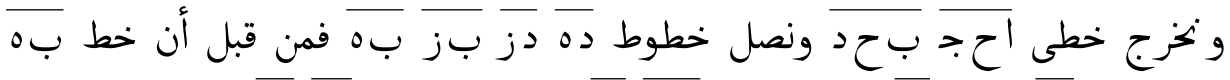

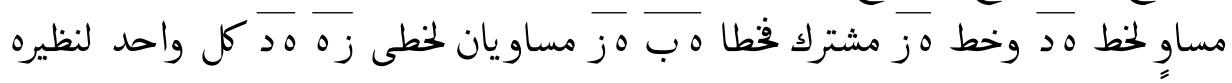

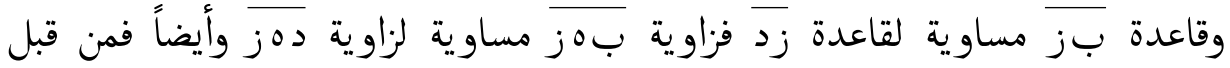
أن خط بـ مساوٍ لخط Io

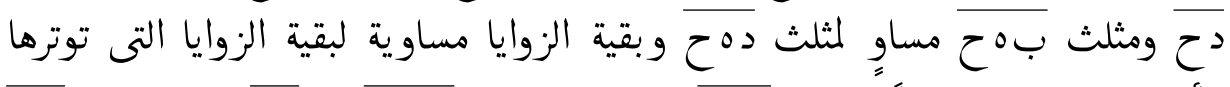

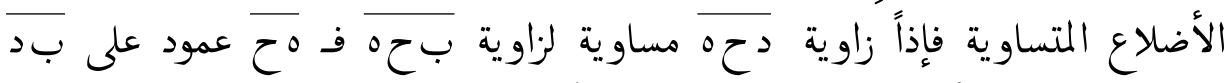

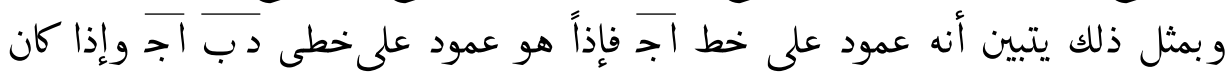

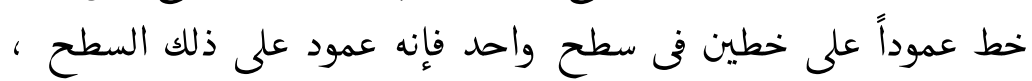

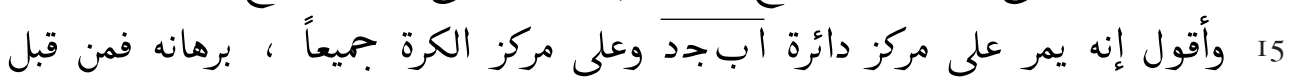

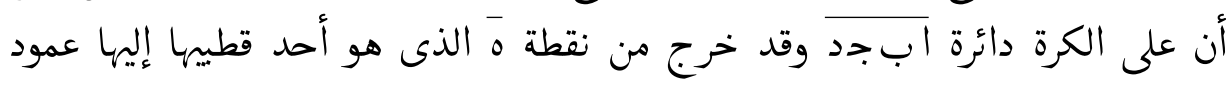

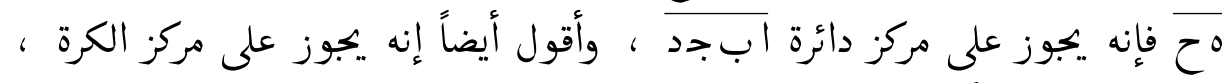

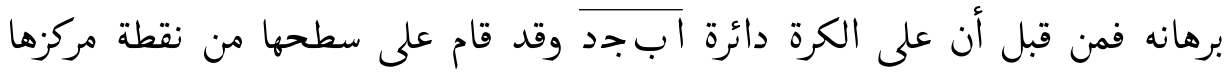

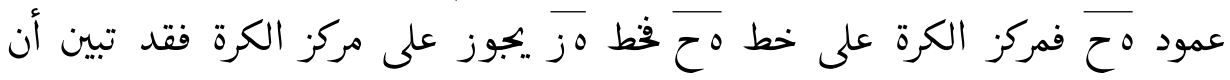
20 أن نبين

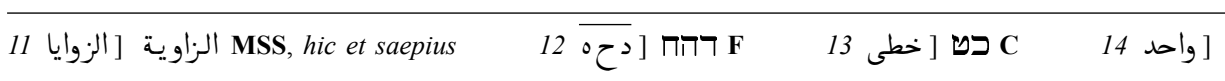
فإنه عمود على خطين في سطح واحد false add. F 
[1r] ] [إذا كانت على كرة دوائر عظام فإنها تنفصل بنصفين .

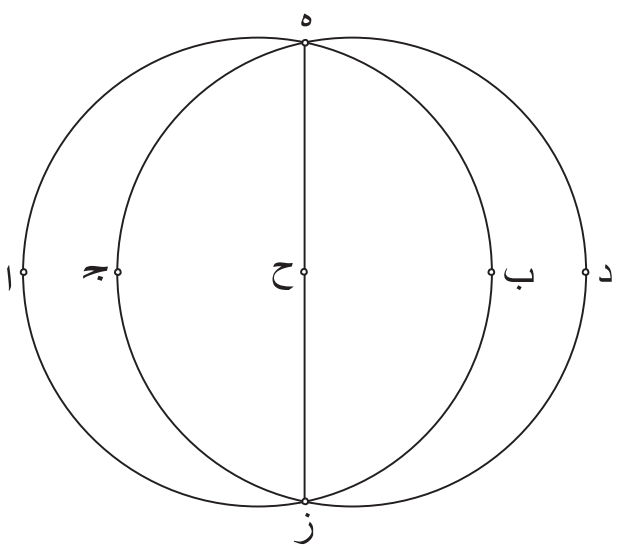

مثال ذلك أنا نفرض على الكرة دوائر عظاهاً وهما دائرتا اب جد تنفصل على نقطتى

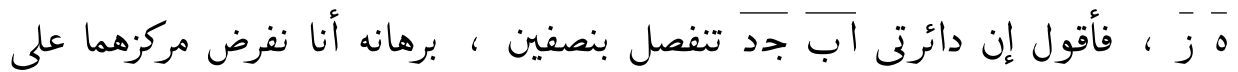

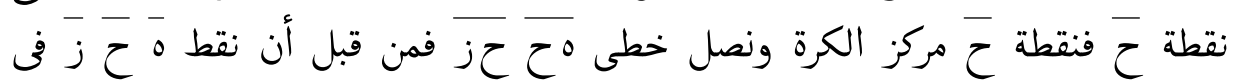
5

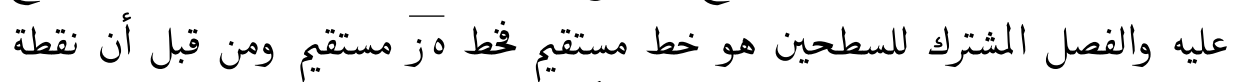

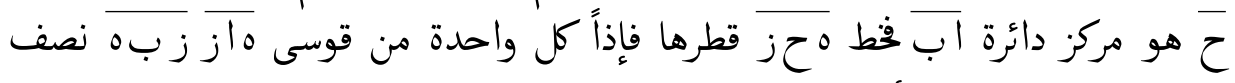

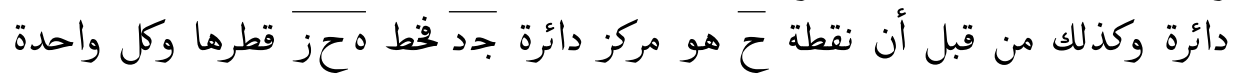

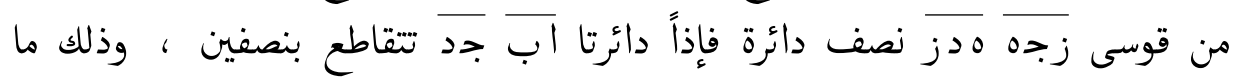

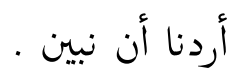

إذا كانت على كرة ما دوائر تنفصل بنصفين فإنها عظام . [ir]

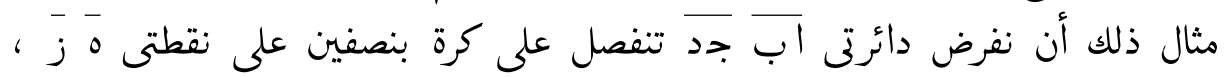

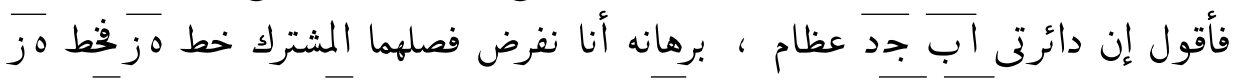

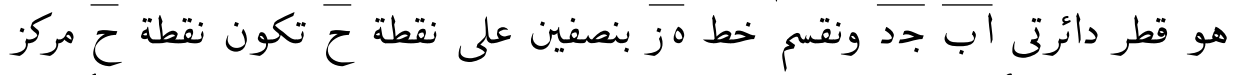
5

1 (Prop. 13)] marg. F 


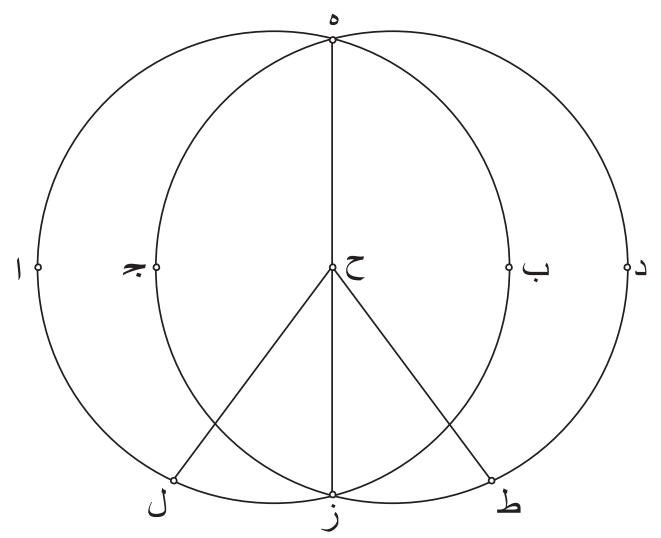

سطح دائرة جد ونفرضه خط حط و نخرج من نقطة ح أيضاً عموداً على سطح

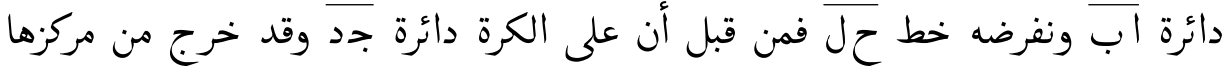

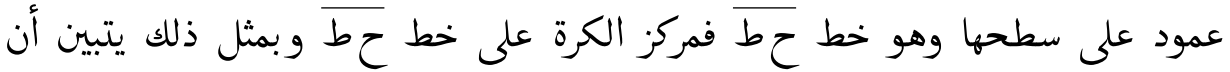

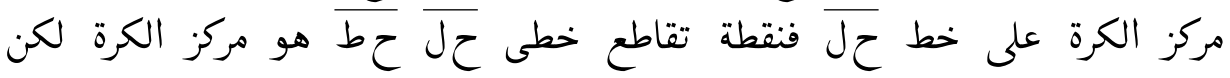

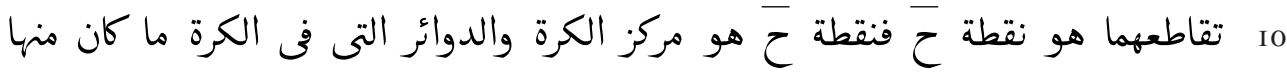

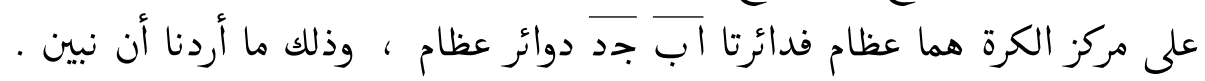
[عا [إذا كانت على كرة دائرة عظيمة وفصلت دائرة أخرى على الكرة على زوايا قائمة

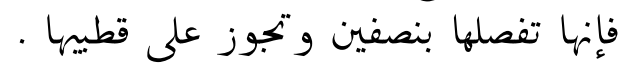

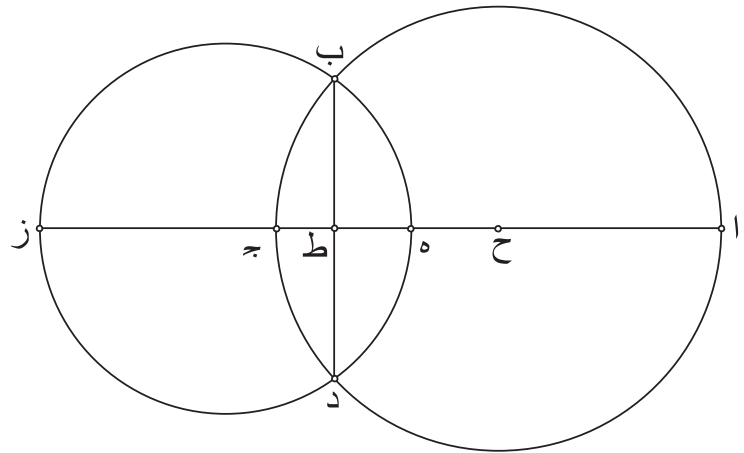

مثال ذلك أنا نفرض دائرة عظيمة على كرة وهى دائرة ابجائد تفصل دائرة أخرى

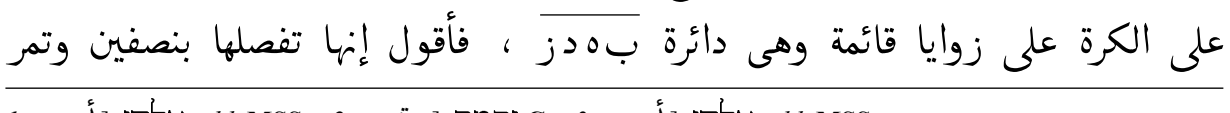

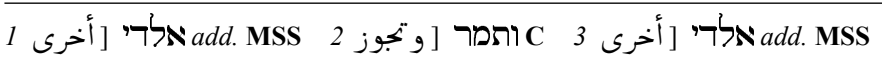




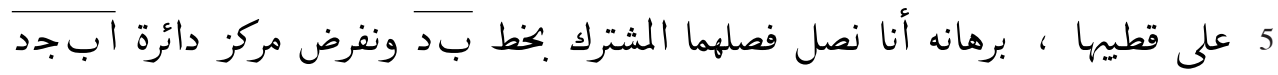

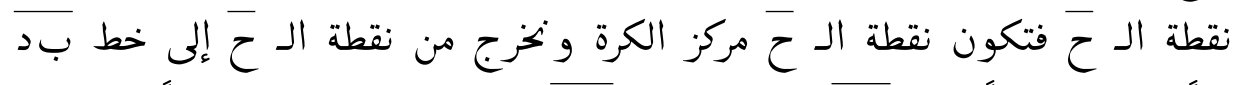

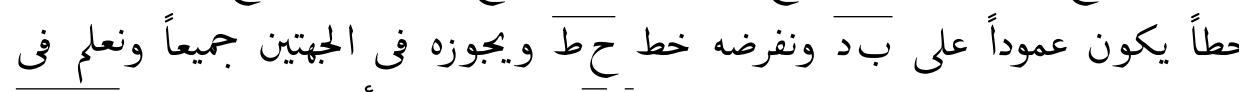

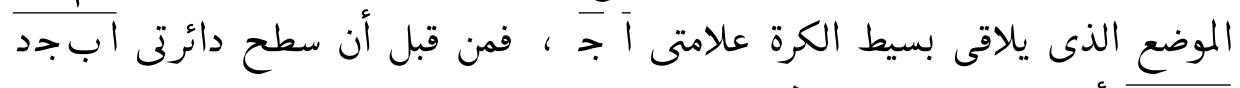

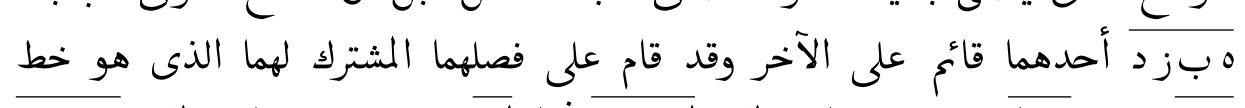
I0

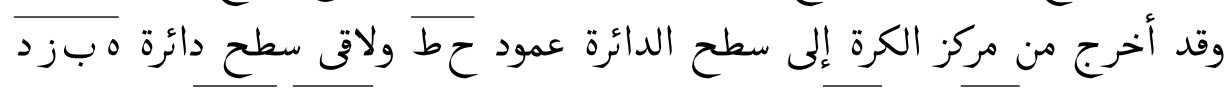

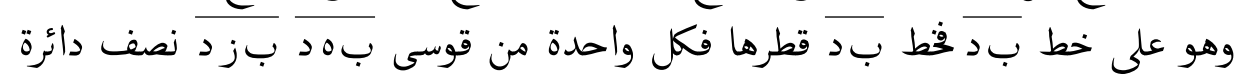

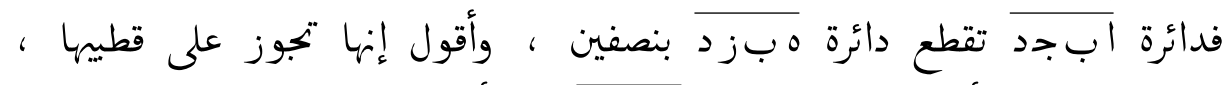

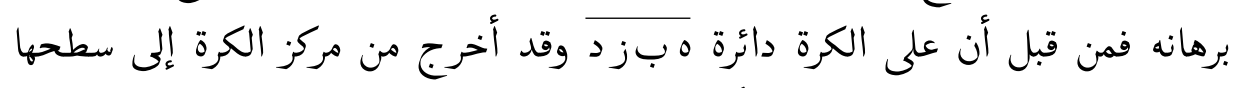

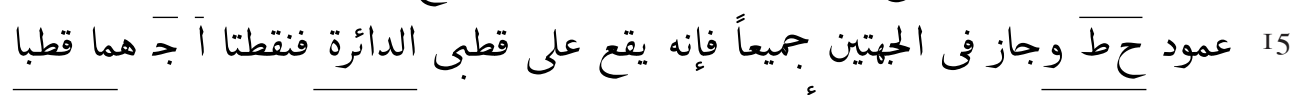

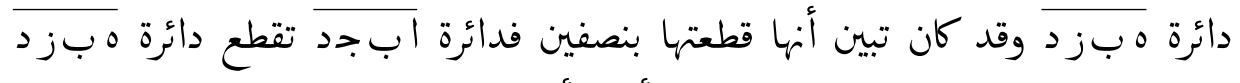

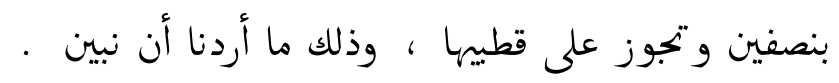

[10] إذا كانت على كرة دائرة عظيمة تقطع دائرة أخرى على الكرة غير عظيمة بنصفين

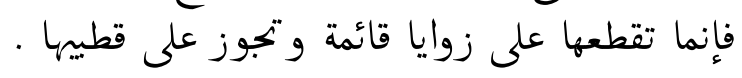

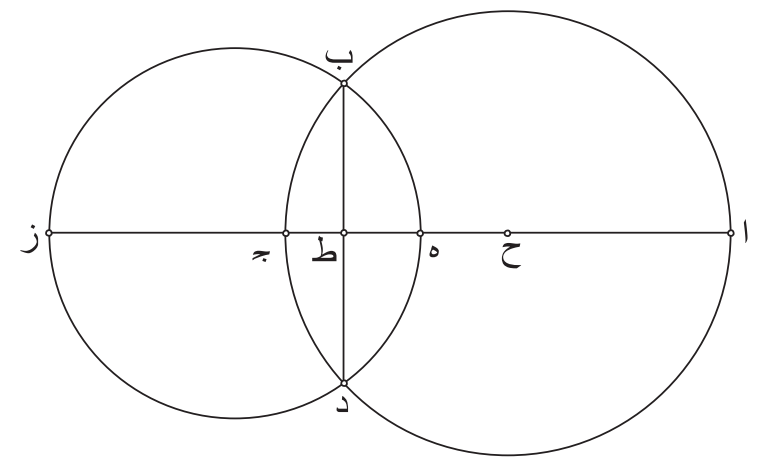

مثال ذلك أنا نفرض دائرة عظيمة على كرة وهى دائرة ابجدد تقطع دائرة أخرى

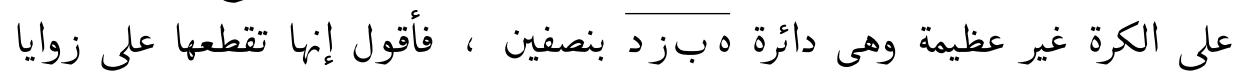

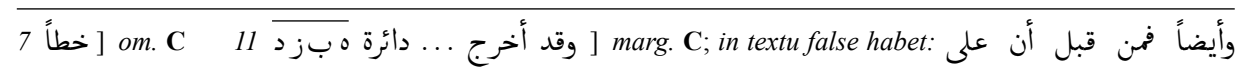

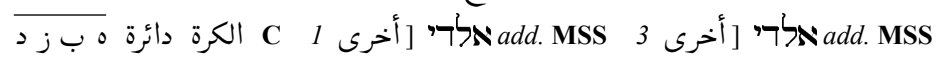


5 قائمة وتجوز على قطيها ، برهانه أنا نفرض فصلهما المشترك لهما خط بـد فمن قبل

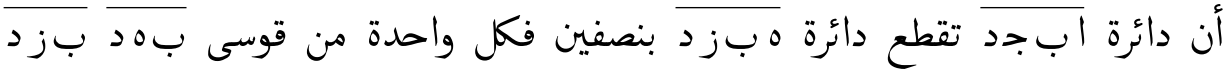

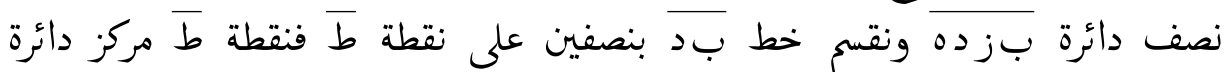

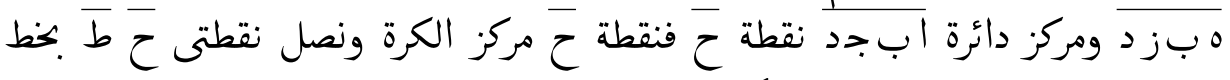

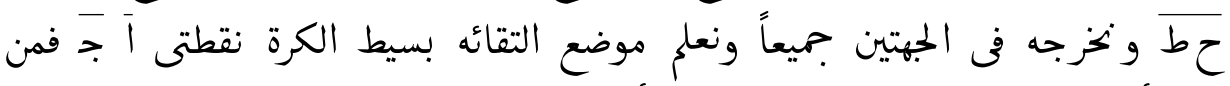

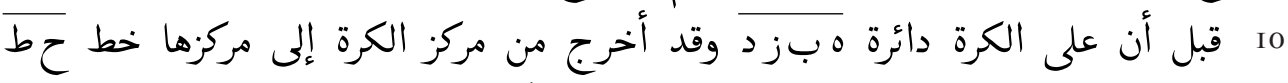

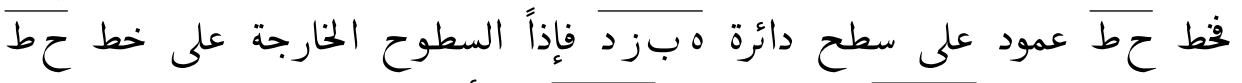

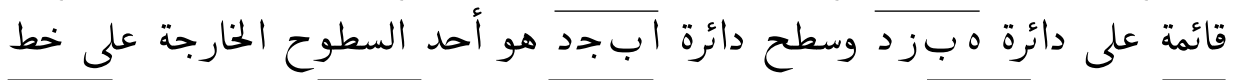

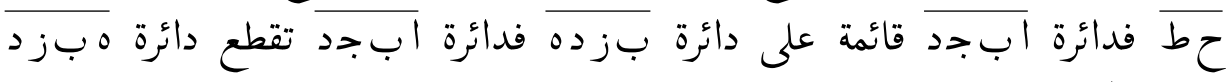

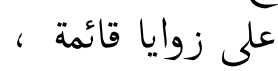

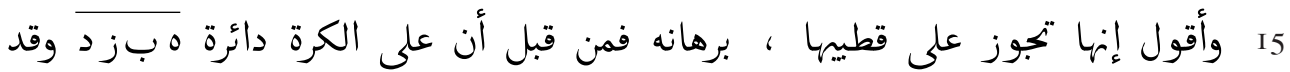

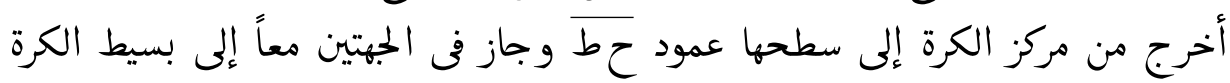

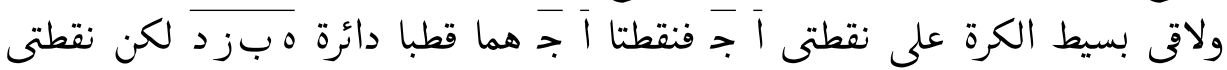

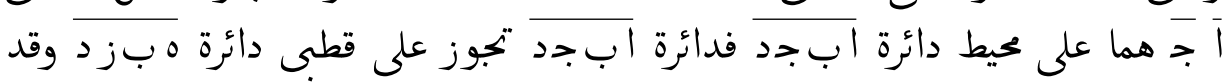

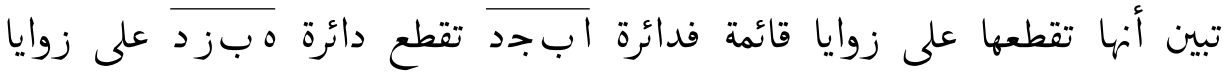

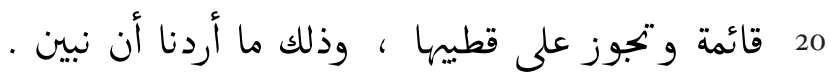

[11] [إذا كانت على كرة دائرة عظيمة تفصل دائرة أخرى على الكرة وتمر على قطييها فإنها

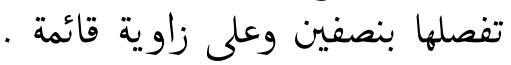

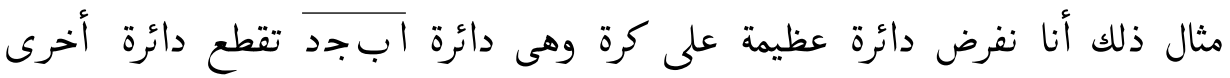

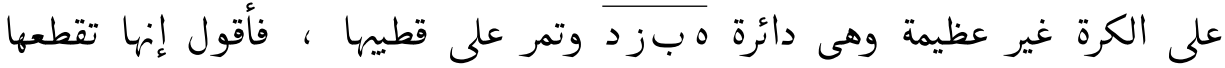

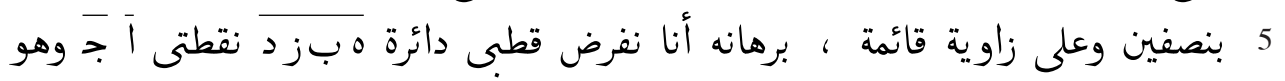

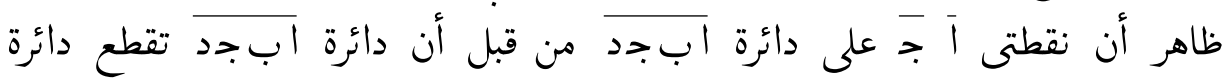

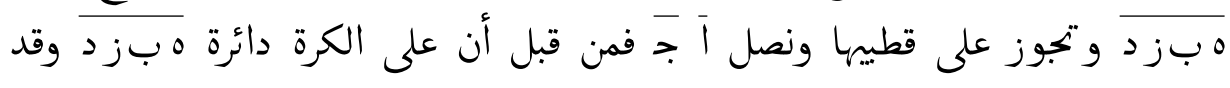

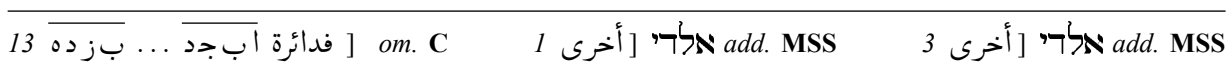

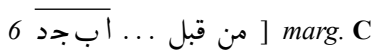




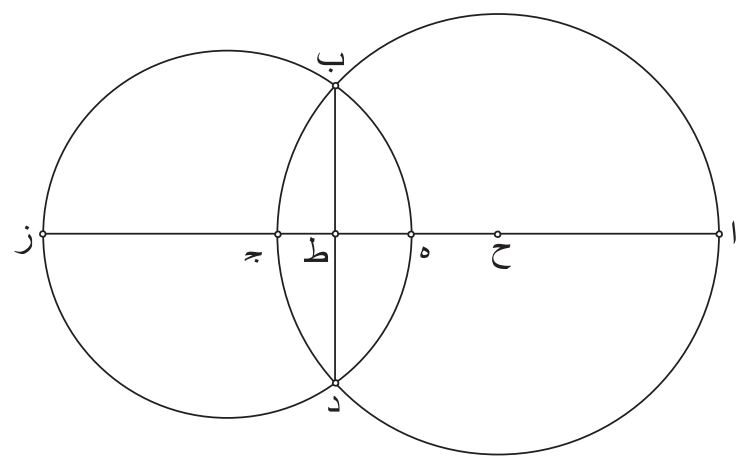

وصل فيما بين قطييها خط اج وإذا كانت دائرة على كرة فإن الخط الذى يصل بين

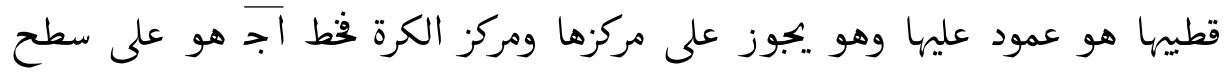

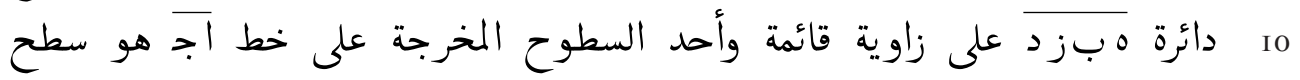

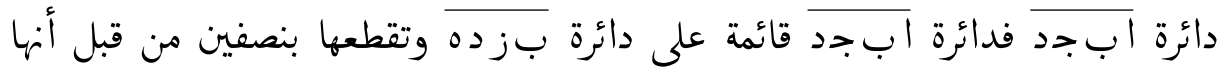

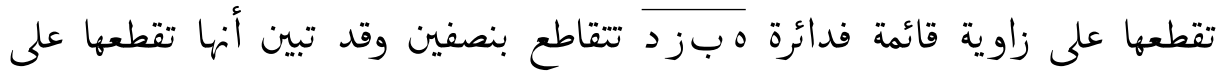

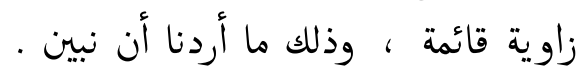

[iv] [إذا كانت دائرة عظيمة على كرة فالخط الخارج من قطبها إلى ميطها مساوٍ لضلع المربع المخطوط فيها - مانت

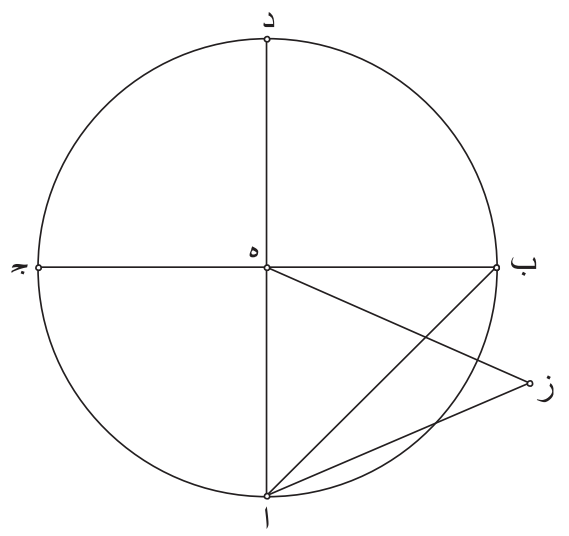

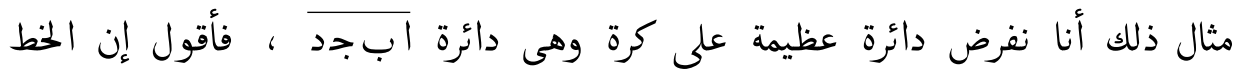

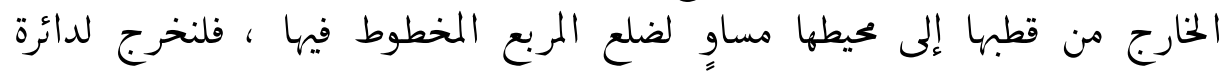
[مال ذلك ... فيها 4-3 repet. et del. F 


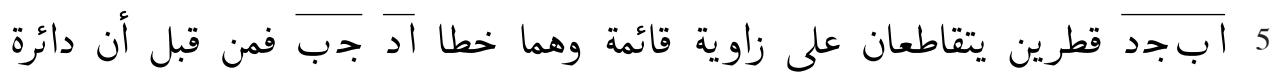

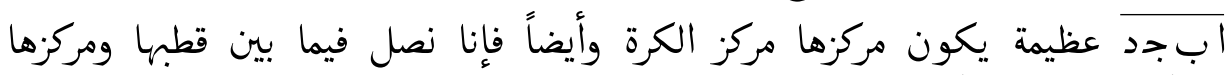

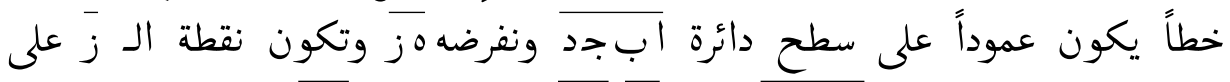

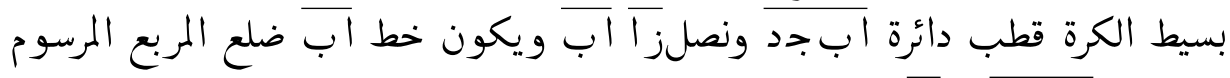

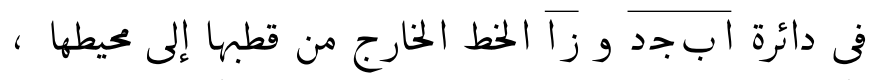

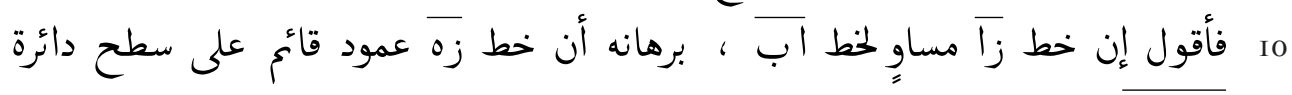

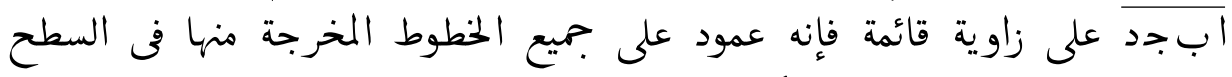

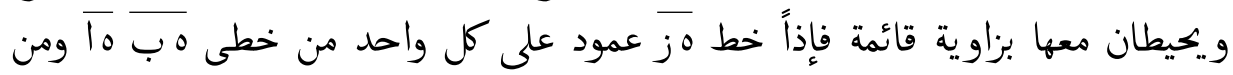

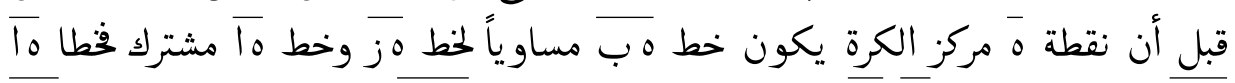

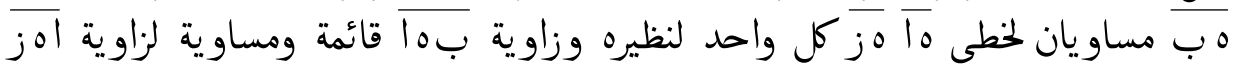

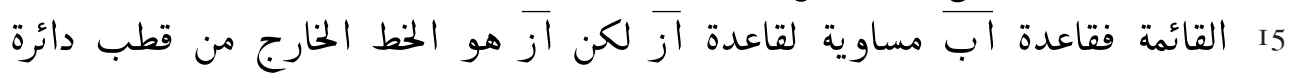

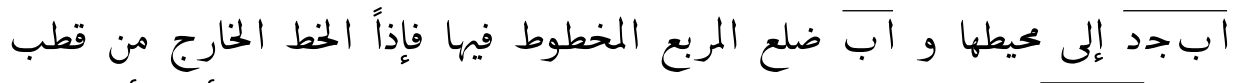

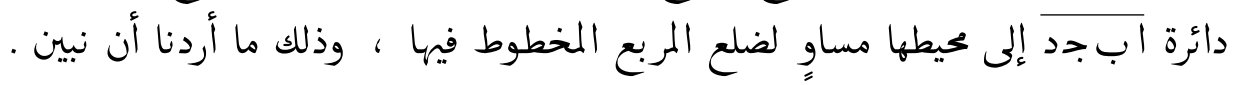
[1،] إذا كانت دائرة على كرة وكان الخط الخارج من قطبها إلى محيطها مساوياً لضلع المربع

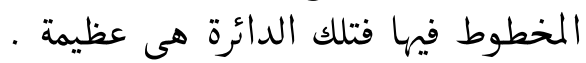

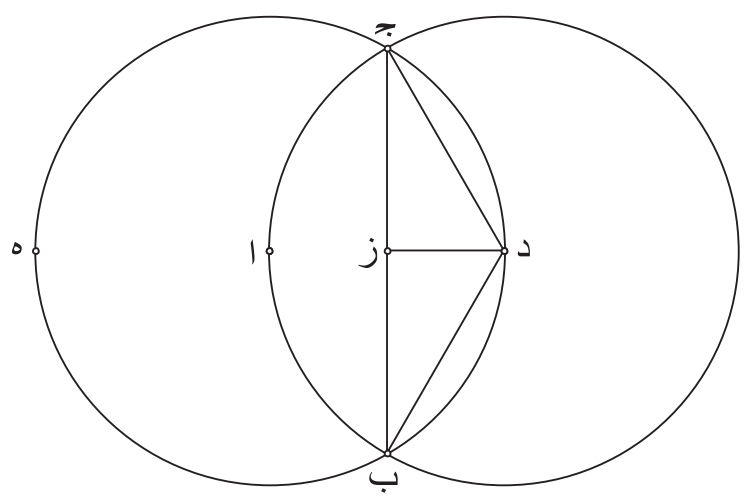

مثال ذلك أن نفرض دائرة ابج ويكون قطبها على نقطة دَ ويكون الخط الخارج

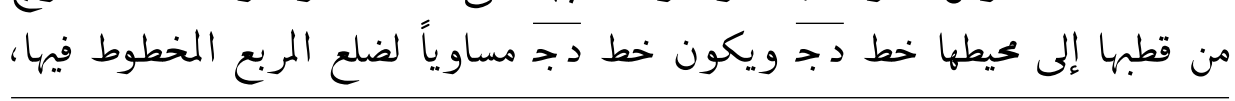
$\overline{7} \overline{j 0}]$ illeg. $\left.\mathbf{C} \quad 8 \overline{j_{j}}\right] \mathbf{C} \mathbf{C}$ 
5 فأقول إن دائرة ابج عظيمة ، برهانه أنا نفرض على خط داج وعلى مركز الكرة

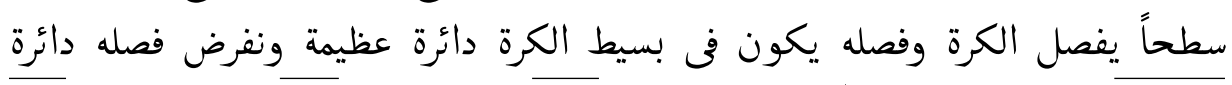

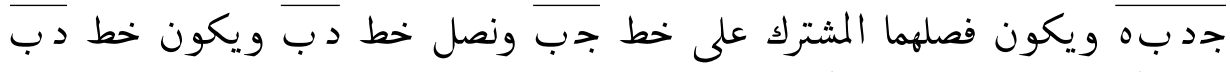

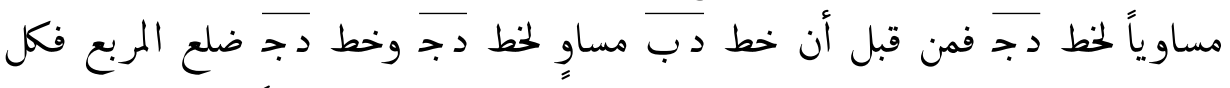

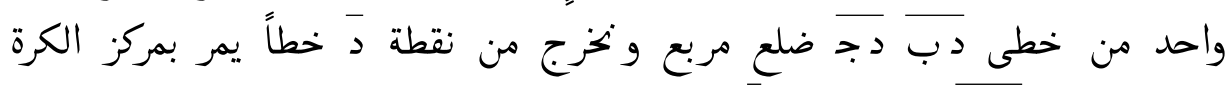

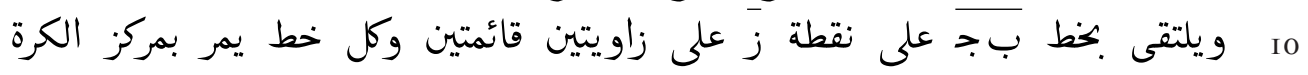

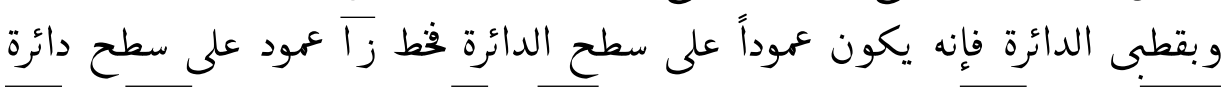

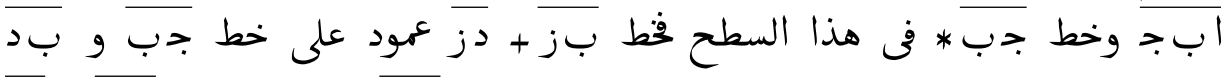

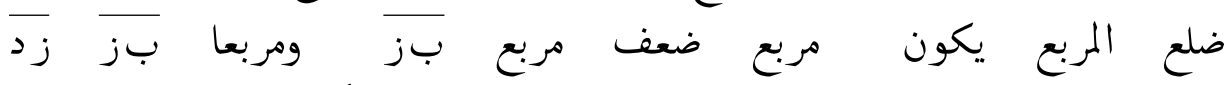

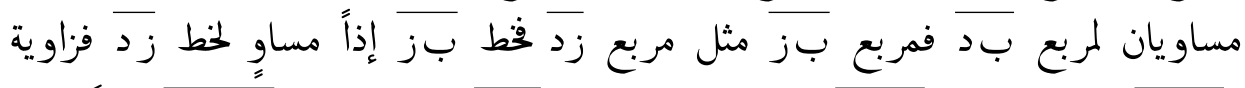
I5

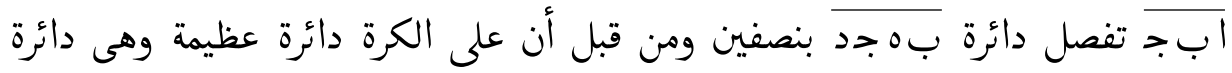

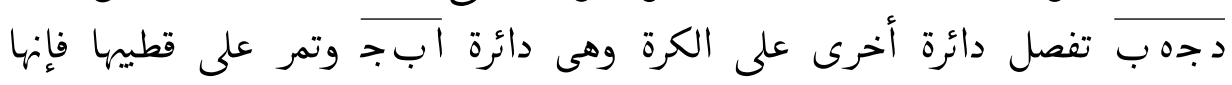

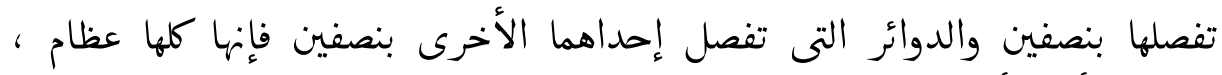
وذلك ما أردنا أن نبين والنوائر

$$
\text { نريد أن نجد قطر دائرة مفروضة على كرة . }
$$

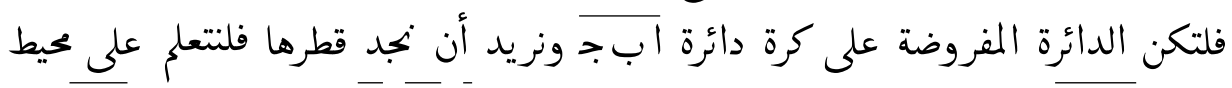

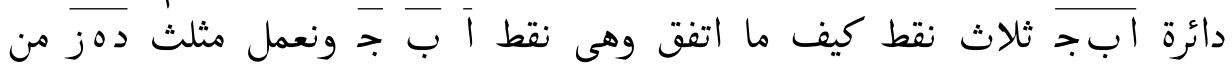

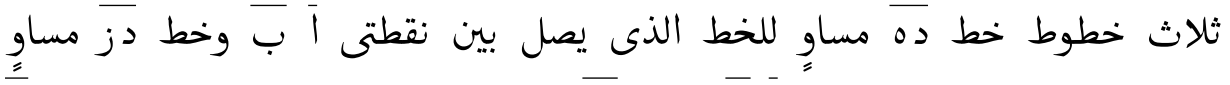
5

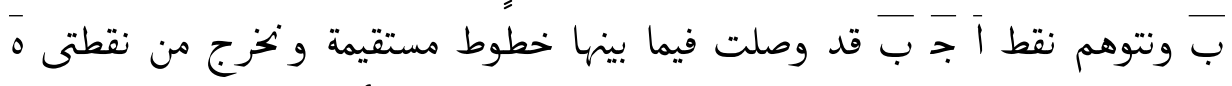

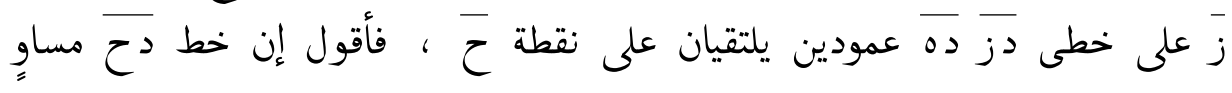

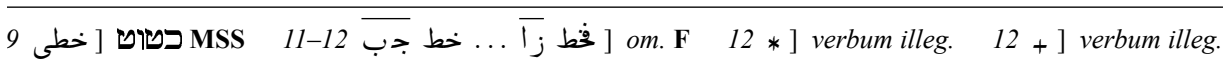

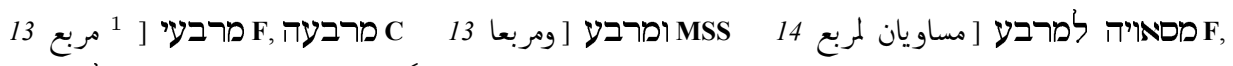
[ فإذاً ... بنصفين 16- om. F 

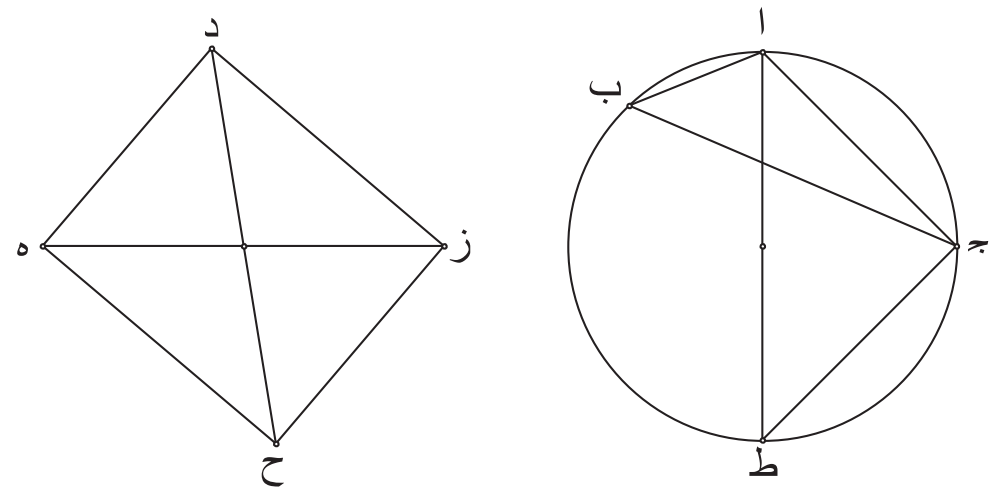

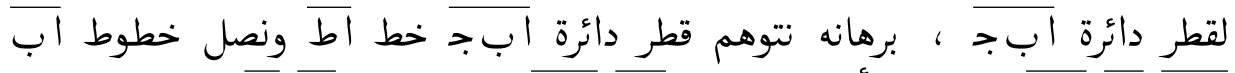

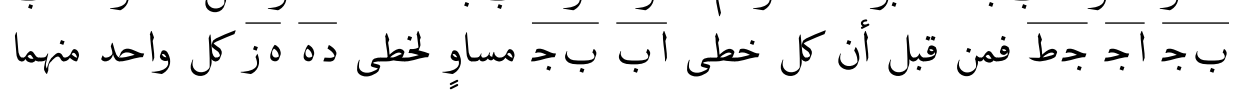

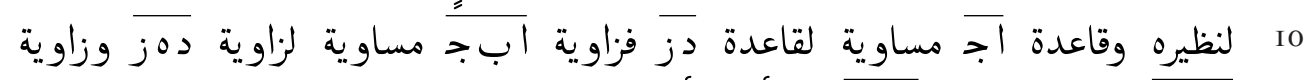

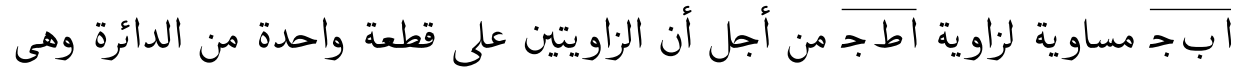

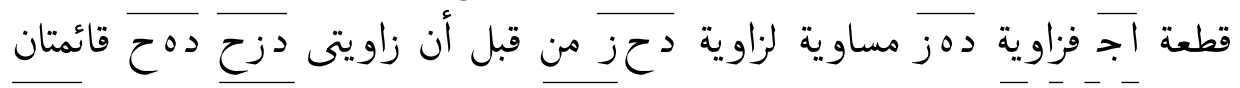

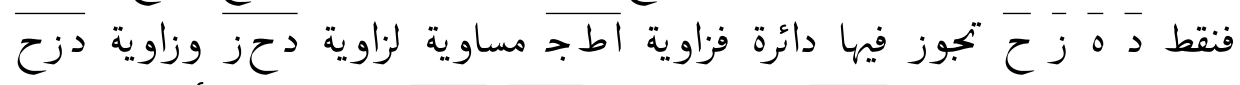

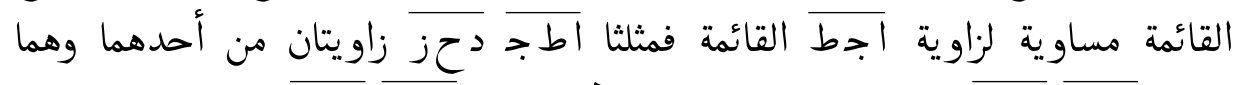
15

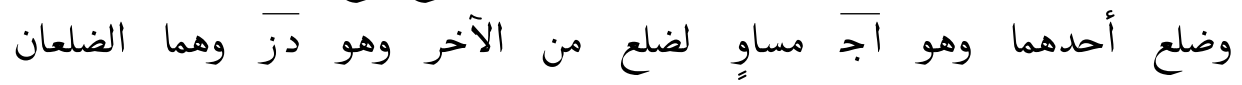

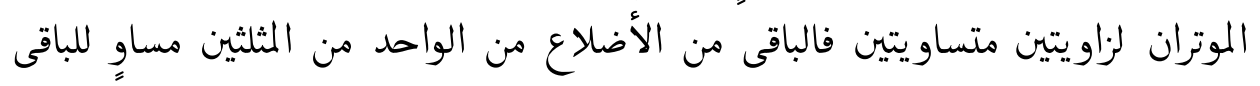

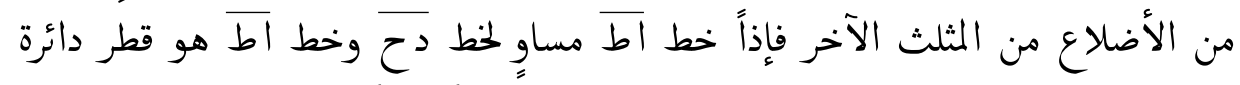

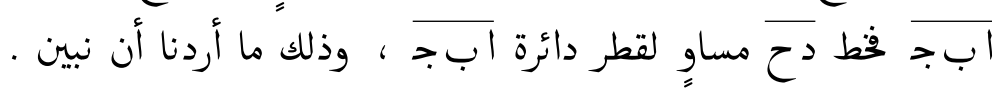

$$
\text { نريد أن نجد قطر كرة مفروضة . }
$$

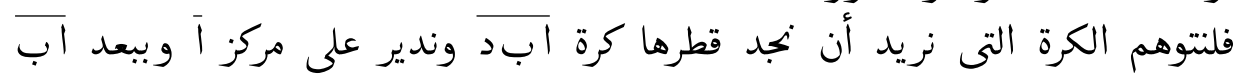

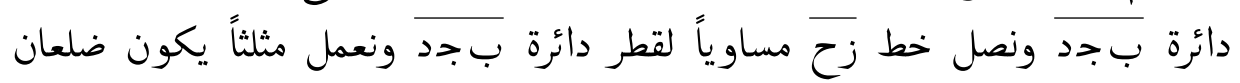

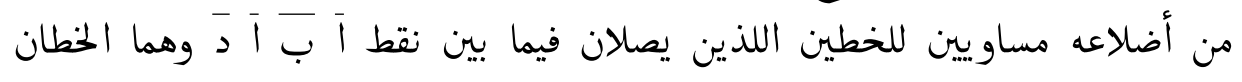

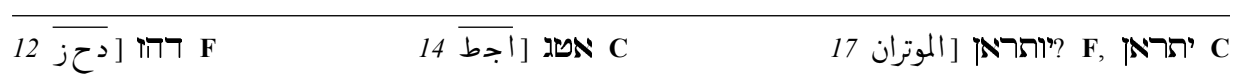

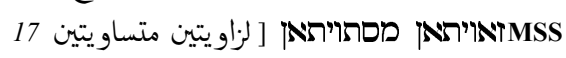



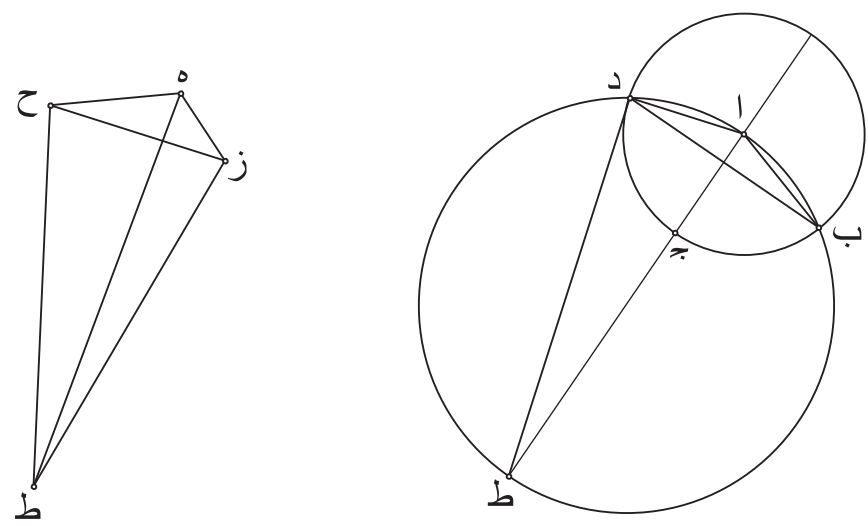

5 الخارجان من قطب دائرة بَجد إلى ميطها والآخر مساوٍ لقطرها ونفرضه مثلث الخدا

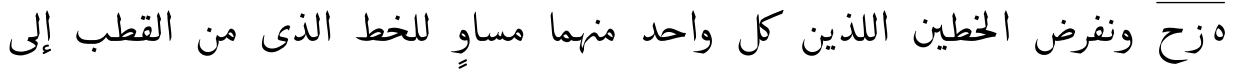
المحيط ضلعى 0ز

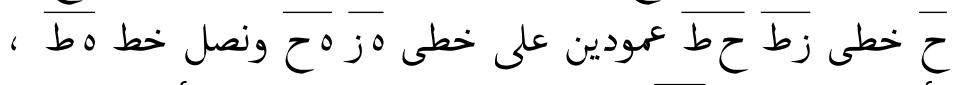

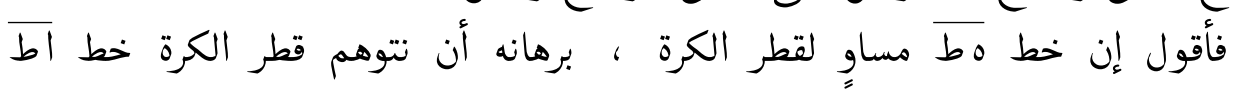
و ونخرج على خط اط سطحاً يفصل الكرة ويكون فصله في بسيط الكرة دائرة إنهرة

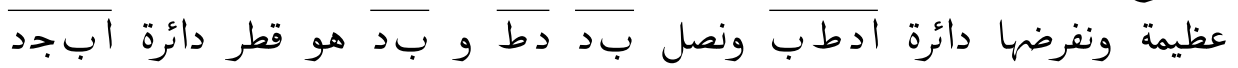

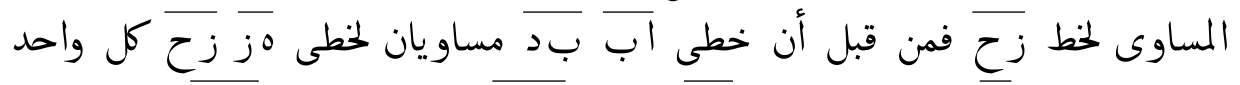

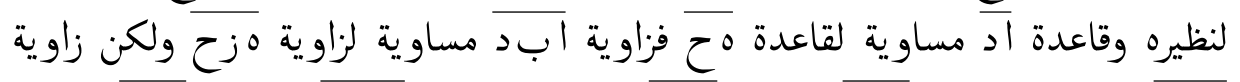

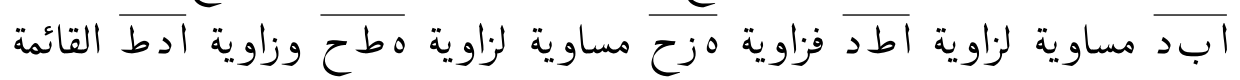

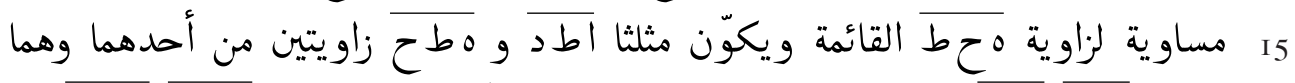

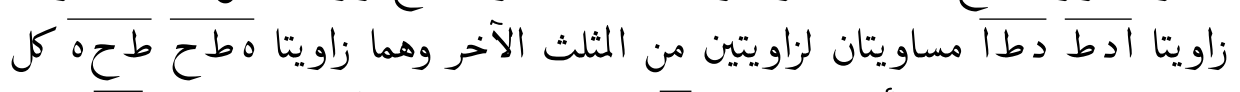

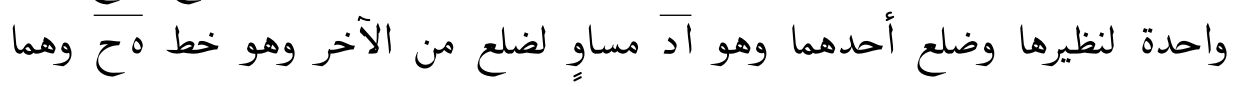

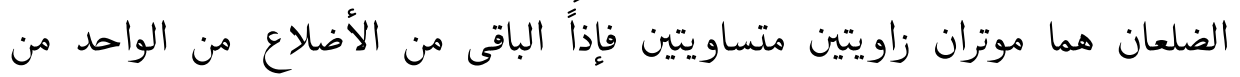

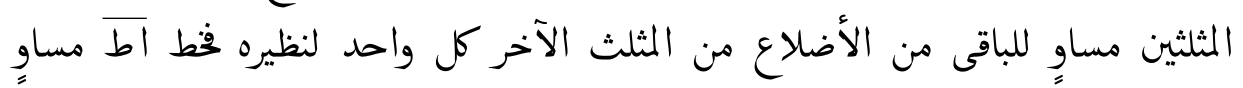
20 أردنا أن نبين مُطط

18-19 om.C 
[r] نريد أن نخط دائرة عظيمة على نقطتين معلومتين على بسيط الكرة .

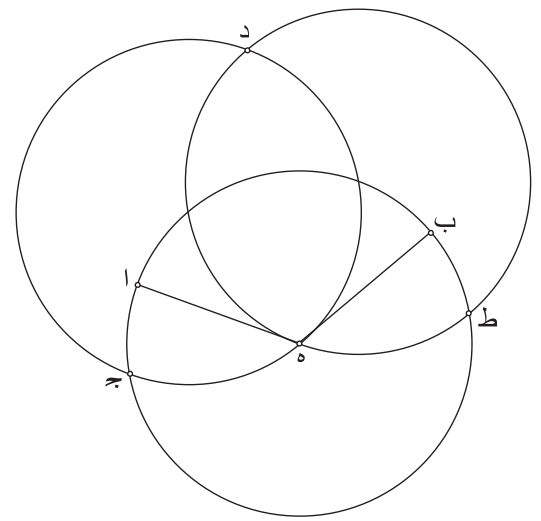

فلتكن النقطتان المعلومتان نقطتى آ بَ ونريد >أن> نخط على نقطتى آ بَ دائرة

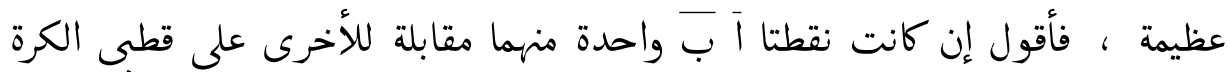

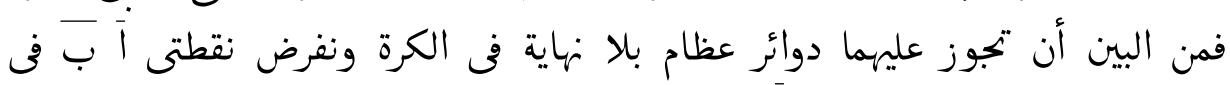

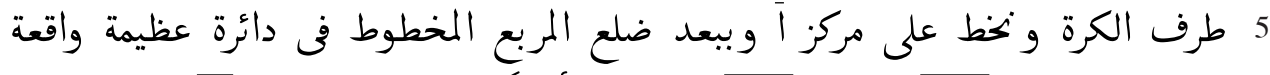

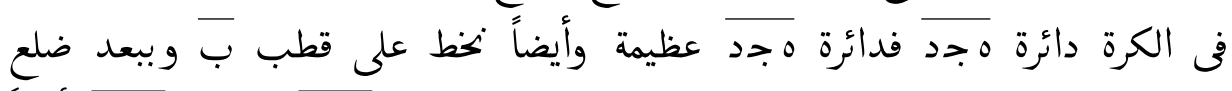

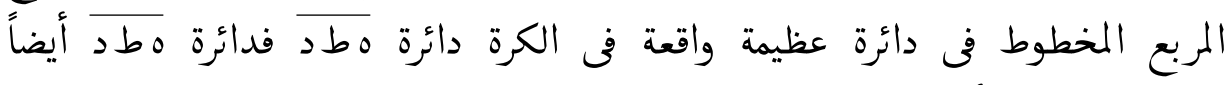

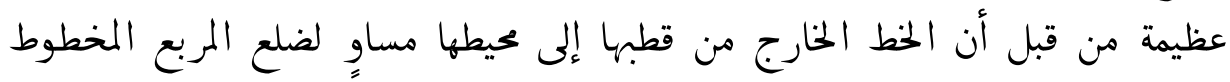

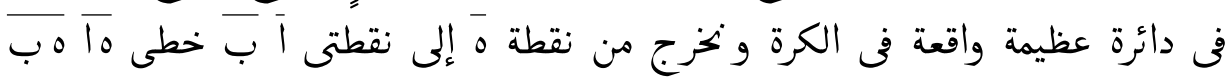

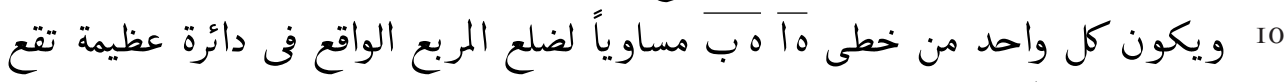

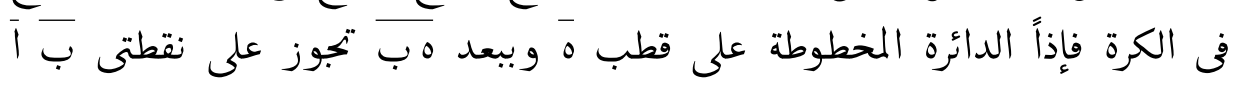

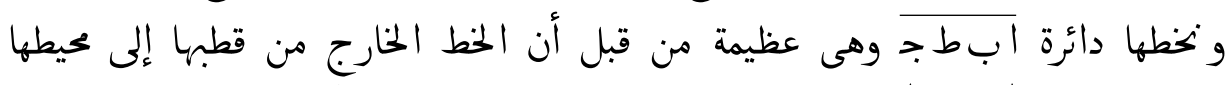

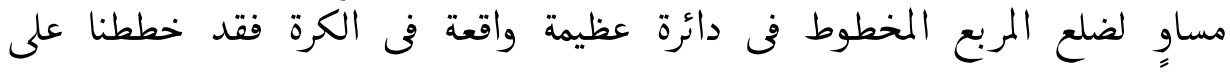

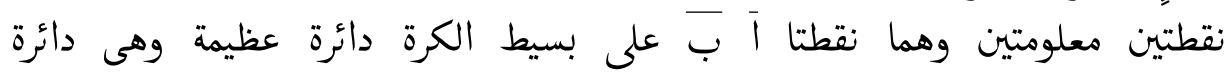

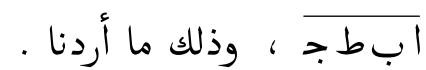

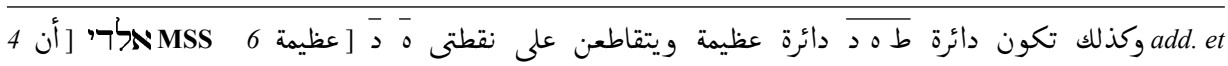

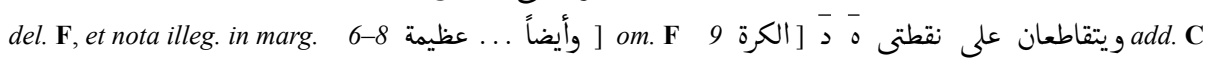


[rr] نريد أن نجد قطب دائرة معلومة على الكرة .

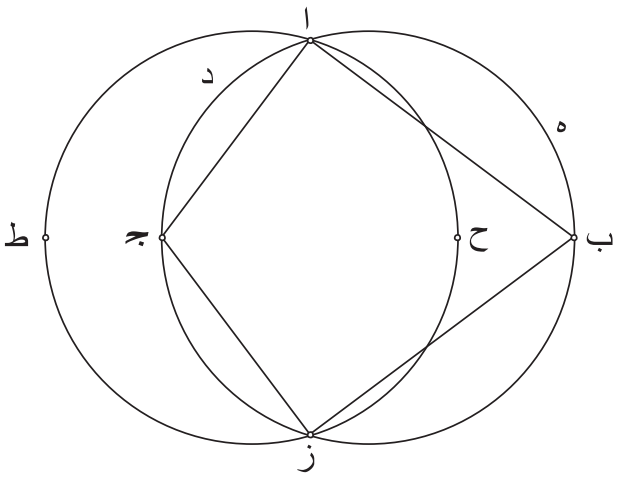

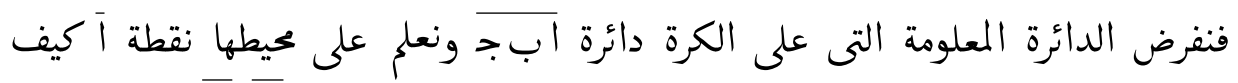

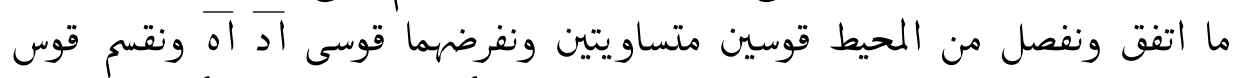

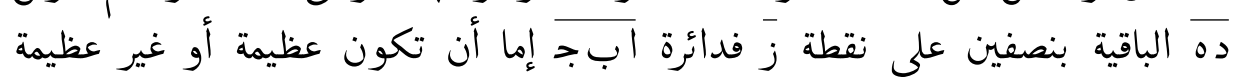

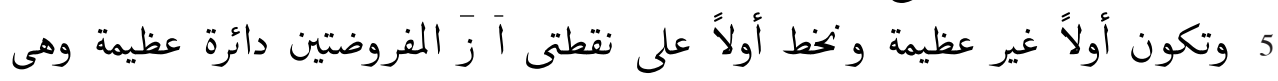

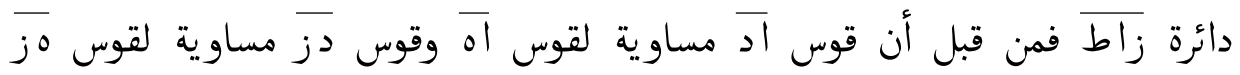

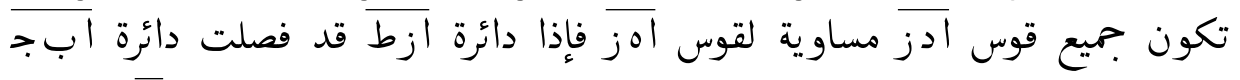

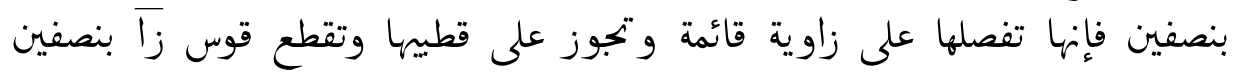

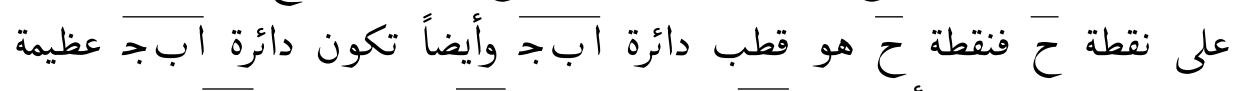

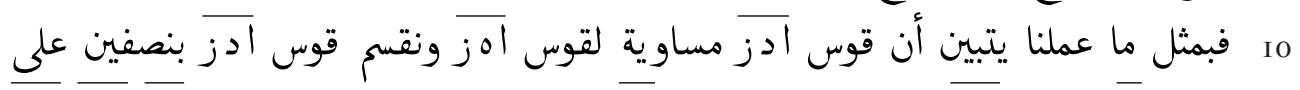

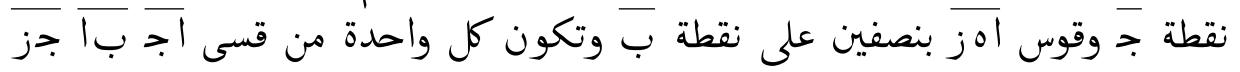

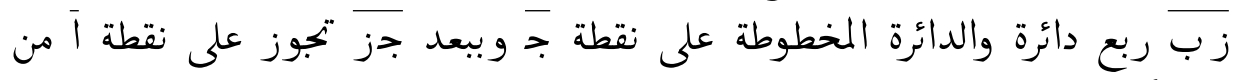

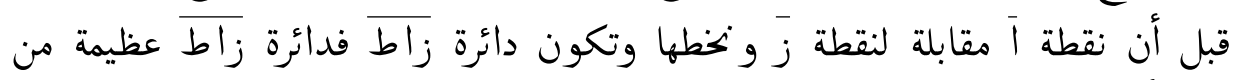

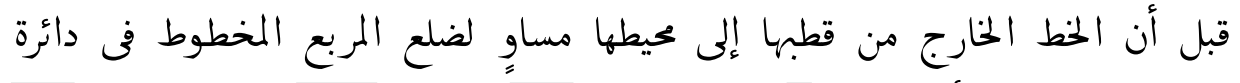

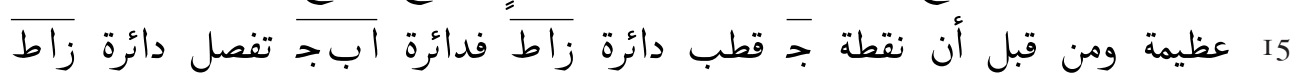

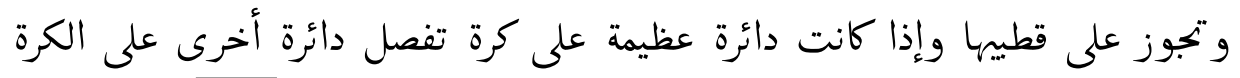

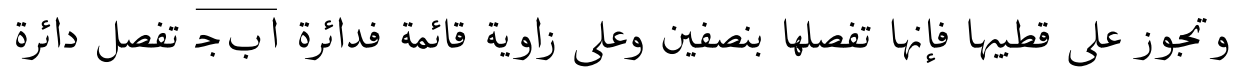

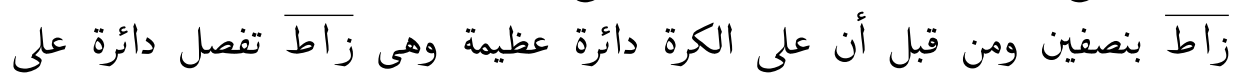


Paul Kunitzsch, Richard Lorch

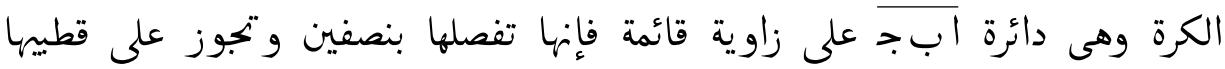

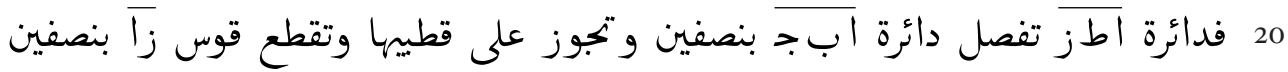

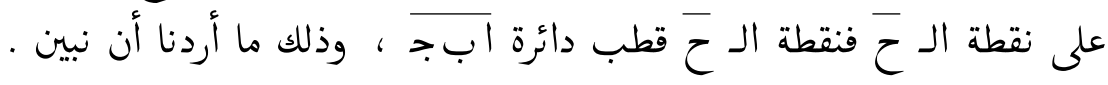

كملت المقالة الأولى [ نبين add.C 